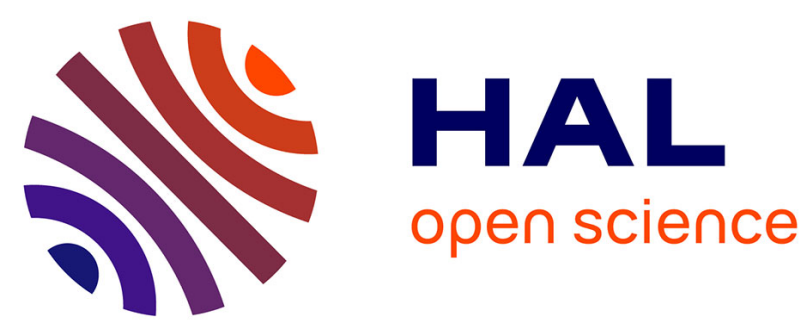

\title{
Voltage Regulation and Current Sharing in DC Microgrids with different Information Scenarios
}

Alessio Iovine, Miguel Jiménez Carrizosa, Elena de Santis, Maria Domenica

Di Benedetto, Pierdomenico Pepe, Alberto Sangiovanni-Vincentelli

\section{- To cite this version:}

Alessio Iovine, Miguel Jiménez Carrizosa, Elena de Santis, Maria Domenica Di Benedetto, Pierdomenico Pepe, et al.. Voltage Regulation and Current Sharing in DC Microgrids with different Information Scenarios. IEEE Transactions on Control Systems Technology, 2022, 10.1109/TCST.2021.3126063 . hal-03452137

\section{HAL Id: hal-03452137}

https://hal-centralesupelec.archives-ouvertes.fr/hal-03452137

Submitted on 26 Nov 2021

HAL is a multi-disciplinary open access archive for the deposit and dissemination of scientific research documents, whether they are published or not. The documents may come from teaching and research institutions in France or abroad, or from public or private research centers.
L'archive ouverte pluridisciplinaire HAL, est destinée au dépôt et à la diffusion de documents scientifiques de niveau recherche, publiés ou non, émanant des établissements d'enseignement et de recherche français ou étrangers, des laboratoires publics ou privés. 


\title{
Voltage Regulation and Current Sharing in DC Microgrids with different Information Scenarios
}

\author{
Alessio Iovine, Member, IEEE, Miguel Jiménez Carrizosa, Elena De Santis, Senior Member, IEEE, \\ Maria Domenica Di Benedetto, Fellow, IEEE, Pierdomenico Pepe, Senior Member, IEEE, \\ Alberto Sangiovanni-Vincentelli, Fellow, IEEE
}

\begin{abstract}
We present a general framework for the control of a Direct Current (DC) microgrid with star topology (a common DC bus) consisting of renewable sources of energy, loads and storage devices connected via step-up and step-down DC/DC converters. The control objective is guaranteeing voltage stability in the DC microgrid while delivering power to the loads and extracting energy efficiently from renewable sources. To verify grid voltage regulation among a number of devices via current sharing, we use a Lyapunov-based Input-to-State Stability (ISS) analysis. We consider three control scenarios: distributed, partially distributed and decentralized according to the amount of information available to the controllers.
\end{abstract}

Index Terms-DC microgrid, Voltage regulation, Current sharing, Nonlinear Control, Lyapunov methods, Large-signal stability analysis, Input-to-State Stability, Backstepping, Renewables, Storage devices, DC/DC converter

\section{INTRODUCTION}

Direct Current (DC) microgrids have attracted significant interest because of their advantages over Alternate Current (AC) grids (see [1], [2]) where renewable energy sources and DC loads (such as LED lighting) are present. DC grids consist of i) renewable energy sources (renewables), in particular photo-voltaic (PV) devices, ii) Hybrid Energy Storage Systems (HESSs) consisting of slow and fast storage devices (batteries or super-capacitors), and iii) loads such as LEDs and electric vehicles that operate in DC. Fast and slow storage devices are usually integrated with the renewables to ensure power availability under any circumstances. Slow storage devices, such as batteries and fuel cells, are used to provide energy when renewables are not available, while fast storage devices such as super-capacitors and flywheels are used to compensate power transient variations in power production or consumption (see [3], [4], [5]). To reduce losses due to high currents and to

A. Iovine was with the Electrical Engineering Computer Sciences (EECS) Department, University of California, Berkeley, USA. He is now with the CNRS and L2S, CentraleSupélec, Université Paris-Saclay, 91192 Gif-sur-Yvette, France. Email: alessio.iovine@12s.centralesupelec.fr , alessio@berkeley.edu

M. Jiménez Carrizosa is with the Centro de Electrónica Industrial, Universidad Politécnica de Madrid, CEI-UPM, Spain. Email: miguel.jimenezcarrizosa@upm.es

E. De Santis, M. D. Di Benedetto and P. Pepe are with the Department of Information Science and Engineering and Mathematics (DISIM), Center of Excellence for Research DEWS, University of L'Aquila, L'Aquila, Italy. Email: \{elena.desantis, mariadomenica.dibenedetto,pierdomenico.pepe\}@univaq.it

A. Sangiovanni-Vincentelli is with the Electrical Engineering Computer Sciences (EECS) Department, University of California, Berkeley, USA. Email: alberto@berkeley.edu add redundancy to the system, multiple slow and fast storage devices are commonly used.

To exchange power in a reliable way, DC grid voltage stability must be guaranteed under adverse circumstances (see [6], [2], [7], [8], [9], [10]). The storage device system is usually controlled in a master-slave or droop control configuration, according to the number of devices that are devoted to voltage stabilization, as described in [10]. Responsibilities are allocated according to the available information. The rigorous analysis of voltage stabilization is an open research problem (see [11], [12], [13], [14], [15], [16], [17], [18], [19], [20], [21]). Current sharing stability results [22], [23], [24] are important in this respect.

In this paper, we analyze the stability of voltage and current dynamics involved in the microgrid. We assume that energy and power levels are decided by higher level controllers (see [10], [25]) and are given as set-points that satisfy power balance [7], [8], [9]. We analyze the short-term stability of the microgrid with respect to the set-points and references chosen to guarantee long-term stability [7], [8]. Further, we introduce a constructive modular stability analysis for a DC microgrid, where sources, loads and slow storage devices act as perturbations on a shared DC voltage bus and fast storage devices are used to stabilize voltage. We obtain this result by using nonlinear control techniques such as backstepping (see [26], [27]), dynamic feedback linearization (see [28], [29]) and Input-to-State Stability (ISS) (see [30], [31], [32], [33]). In our stability analysis, we consider a scenario where complete information about the system is available and one where only partial information is provided.

We extend the analysis of a DC microgrid with a single super-capacitor introduced in [4] and [18]. The master-slave distributed nonlinear control technique presented in [4] is modified into a droop-like current sharing one, which is obtained from an ISS Lyapunov function (see [31], [34], [35], [36], [37]) with respect to a reference signal playing the role of a (fictitious) input [38]. The novelty of our results as compared to contributions on current sharing for DC microgrids is the use of a droop-like approach that is closer to well-known control techniques for power systems. We use modularity to facilitate the extension of this approach to the case where the microgrid includes any number of devices.

We verify the stability of customized DC microgrids with a star topology that include devices with non-minimum phase for DC bus voltage regulation, such as step-up converters (see [39]). This case has not been considered in the literature (see 
[16], [22], [24], [40]) even if it is the solution of choice, given that it can use less expensive storage devices. Also in this case, we investigate the cases of complete and partial knowledge of the system by the control subsystem. The resulting Lyapunov-based control laws allow current (and consequently power) sharing by the different devices with the same goal of stabilizing the grid while considering nonlinearities and different communication topologies. The literature considers mostly linear techniques with distributed communication (see [41]). We show that our control laws are less complex and consequently easier to implement with respect to previous results (see [4]). We generalise specific solutions adopted for particular microgrids (see [18], [38], [42]). Finally, we distribute tasks to the microgrid devices for steady-state stability using a Lyapunov ISS approach.

The paper is organized as follows. Section III introduces the model of the DC microgrid. In Section IV, we present the control laws for each subsystem, while Section V provides Lyapunov functions for stability analysis. In Section VI, we show that the entire system is stable using the results of Section IV. Section VII provides simulation results, and Section VIII offers conclusions.

\section{NOTATION AND BASIC DEFINITIONS}

The symbol $|\cdot|$ denotes the absolute value, the symbol $\|\cdot\|$ denotes the Euclidean norm and $\|\cdot\|_{p}$ denotes the p-norm in $\mathbb{R}^{n}$. The symbol $\circ$ denotes composition (of functions). The notation $\dot{f}$ and $\ddot{f}$ indicate the first and second derivative, respectively, of the function $f$ with respect to time. Given a finite set $S$, its cardinality is denoted as $\operatorname{card}(S)$. The operator col produces a single column vector composed by the the aggregation of other vectors. That is, given $m$ vectors $s_{i} \in \mathbb{R}^{n}$, $i=1, \ldots, m$, the resulting vector $s=\operatorname{col}\left[s_{i}\right], i=1,2, \ldots, m$, is:

$$
s=\operatorname{col}\left[s_{i}, i=1, \ldots, m\right]=\left[\begin{array}{llll}
s_{1}^{T} & s_{2}^{T} & \ldots & s_{m}^{T}
\end{array}\right]^{T} \in \mathbb{R}^{n m} .
$$

We refer to [26] for the definition of Lyapunov functions, and functions $\mathcal{K}, \mathcal{K}_{\infty}$ and $\mathcal{K} \mathcal{L}$. We refer to [27], [30], [31], [33], [43], for the definition of Input-to-State Stability and practical Input-to-State Stability.

Given that the argument of all functions in this paper is time, for the sake of notational simplicity, time dependence is omitted.

\section{MICROGRID CONFIGURATION}

A typical DC microgrid consists of a set of devices, as renewables, storage devices, and loads. It is intended to:

I) Extract renewable power and store it,

II) Feed the loads, and

III) Ensure power quality by DC bus voltage regulation.

To achieve these goals, a DC/DC converter is connected to each physical device, providing the degree of freedom to control the device. To satisfy I), the renewables have to be controlled so that the maximum available power is extracted, where the slow storage devices act as a buffer between the power flow requested by the load and the power flow supplied by the renewables. For II), the loads are fed in a Constant
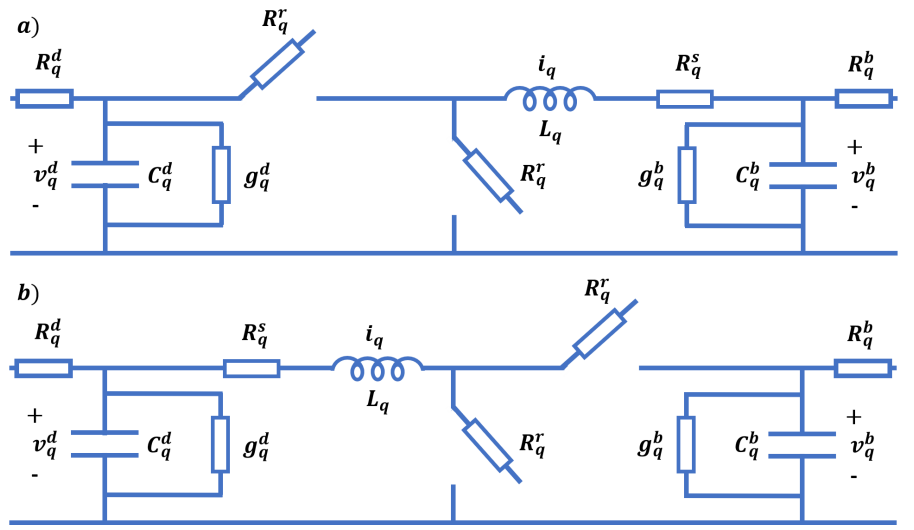

Fig. 1. The electrical scheme of the bidirectional converters: step-down in a) and step-up in b). When unidirectional, the switches in the upper positions are replaced by diodes, but the notation and the mathematical modeling remain the same. On the right hand side, the converters are connected to the DC bus, while on the left hand side they are connected to an exogenous input (voltage source, current source, CIL or CPL).

Power Load (CPL) and Constant Impedance Load (CIL) mode. Finally, the fast storage devices have to be controlled so that voltage regulation and an appropriate transient are obtained (see [7]).

Different types of stability problems arise in a microgrid [7, 8, 9]. We consider in this paper: i) long-term stability, which is ensured by matching the demanded power (loads) with the supplied one provided by renewables and storage devices, and ii) short-term stability, which is related to fast dynamics acting on the DC bus voltage caused by temporary power balance mismatch [7], [8]. Long-term stability is achieved by high-level controllers that provide the power references for the supplied power such that the loads are fed while ensuring a minimum level of charge in the storage devices. Short-term stability is achieved by local controllers that deal with fast variations of loads, renewable power and disturbances [7], [10], [25].

In the sequel, we make the following assumptions:

Assumption 1. The components in the DC microgrid always provide the power demanded by the loads.

Assumption 2. Higher-level controllers provide constant voltage, currents, or power references/set-points for the local controllers. The set-points are such that power balance in steady-state is achieved (see [7]).

Assumption 3. The voltages and currents of the sources and loads and their time derivatives are bounded over time.

\section{A. Microgrid Modeling}

Let $\mathcal{I}_{\eta}$ be the index set corresponding to the renewables, the loads and the slow storage devices, and let $\mathcal{I}_{\phi}$ be the index set corresponding to the fast storage devices. Each element indexed by the sets $\mathcal{I}_{\eta}$ and $\mathcal{I}_{\phi}$ is connected to the DC grid by a DC/DC converter. The mathematical models used here are obtained using the power electronics averaging technique with Pulsed Width Modulation (PWM) [44]. We 
TABLE I

STATE SPACE MATRICES OF THE CONVERTERS

\begin{tabular}{|c|c|c|c|c|c|c|c|c|}
\hline \multirow{2}{*}{$\begin{array}{l}\text { Converter type } \\
\text { a) } \\
\text { step-down source- } \\
\text { bus; step-up load-bus }\end{array}$} & \multicolumn{3}{|c|}{$A_{q}$} & \multicolumn{2}{|l|}{$G_{q}$} & \multicolumn{3}{|c|}{$B_{q}$} \\
\hline & $\begin{array}{c}-g_{q}^{d}\left(C_{q}^{d}\right)^{-1}-\delta_{q} \\
0 \\
0\end{array}$ & $\begin{array}{c}0 \\
-g_{q}^{b}\left(C_{q}^{b}\right)^{-1}-\left(R_{q}^{b} C_{q}^{b}\right)^{-1} \\
-L_{q}^{-1}\end{array}$ & $\begin{array}{c}-C_{q}^{d^{-1}} \\
C_{q}^{b^{-1}} \\
-\left(R_{q}^{s}+R_{q}^{r}\right) L_{q}^{-1} \\
\end{array}$ & {$\left[\begin{array}{c}0 \\
\left(R_{q}^{b} C_{q}^{b}\right)^{-1} \\
0\end{array}\right]$} & {$\left[\begin{array}{c}\delta_{q}^{d} \\
0 \\
0\end{array}\right]$} & & $\begin{array}{l}0 \\
0 \\
L_{q}^{-1} \\
\end{array}$ & $\left.\begin{array}{c}C_{q}^{d^{-1}} \\
0 \\
0\end{array}\right]$ \\
\hline $\begin{array}{l}\text { b) } \\
\text { step-up source-bus; } \\
\text { step-down load-bus }\end{array}$ & $\begin{array}{c}-g_{q}^{d}\left(C_{q}^{d}\right)^{-1}-\delta_{q} \\
0 \\
L_{q}^{-1}\end{array}$ & $\begin{array}{c}0 \\
-g_{q}^{b}\left(C_{q}^{b}\right)^{-1}-\left(R_{q}^{b} C_{q}^{b}\right)^{-1} \\
0\end{array}$ & $\begin{array}{c}-C_{q}^{d^{-1}} \\
C_{q}^{b^{-1}} \\
-\left(R_{q}^{s}+R_{q}^{r}\right) L_{q}^{-1}\end{array}$ & {$\left[\begin{array}{c}0 \\
\left(R_{q}^{b} C_{q}^{b}\right)^{-1} \\
0\end{array}\right]$} & {$\left[\begin{array}{c}\delta_{q}^{d} \\
0 \\
0\end{array}\right]$} & {$\left[\begin{array}{l}0 \\
0 \\
0\end{array}\right.$} & $\begin{array}{r}0 \\
0 \\
-L_{q}^{-}\end{array}$ & $\left.\begin{array}{c}0 \\
-C_{q}^{b^{-1}} \\
0\end{array}\right]$ \\
\hline
\end{tabular}

use the standard Quasi-Stationary Line (QSL) approximation of the power lines, as in [17], and [22].

The electrical scheme of each bidirectional step-up or stepdown converter is shown in Fig. 1. In the case of unidirectional converters, as for renewables or loads, a diode replaces a switch, without changing the mathematical description (see [44] for more details). Each converter is indexed with $q \in$ $\left\{\mathcal{I}_{\eta} \cup \mathcal{I}_{\phi}\right\}$. The symbols $v_{q}^{d}, v_{q}^{b}$ and $i_{q}$ denote the voltages at the input and output capacitors, and of the currents through the inductors, respectively. We assume $v_{q}^{d}, v_{q}^{b}>0$, while $i_{q}$ can assume both positive and negative values. On the righthand side, the converters are connected to the DC bus $v_{b u s}$, while on the left-hand side, they are connected to an exogenous input $\delta_{q}^{d}$ (voltage source, current source, Constant Power Load (CPL) or Constant Impedance Load (CIL)). In the sequel, we use the subscripts for describing to which set of converters the variables take part (or their union, in case), while we use superscripts to address the difference between the voltages of the capacitances connected to the DC bus, i.e. $v_{q}^{b}$, and the voltages connected to the exogenous disturbance inputs, i.e. $v_{q}^{d}$. The same apply to the circuit elements. Here $L_{q}$, and $C_{q}^{d}, C_{q}^{b}$, and $R_{q}^{b}, R_{q}^{d}, g_{q}^{b}, g_{q}^{d}, R_{q}^{r}, R_{q}^{s}$ are known positive values describing the inductors, capacitors and resistances in the circuits, respectively. $R_{q}^{r}$ and $R_{q}^{s}$ are the internal resistance of the switches and of the inductance, respectively.

The state of each converter is denoted by

$$
\begin{aligned}
& \eta_{j}=\left[\begin{array}{lll}
v_{j}^{d} & v_{j}^{b} & i_{j}
\end{array}\right]^{T} \in \mathbb{R}^{3}, \quad \forall j \in \mathcal{I}_{\eta} \\
& \phi_{l}=\left[\begin{array}{lll}
v_{l}^{d} & v_{l}^{b} & i_{l}
\end{array}\right]^{T} \in \mathbb{R}^{3}, \quad \forall l \in \mathcal{I}_{\phi} .
\end{aligned}
$$

Here, the vector $\eta$ includes the voltages and currents of the $n_{\eta}$ converters connected to the $n_{\eta}$ renewables, loads, either for CIL or CPL, and slow storage devices needed to provide longterm stability. The vector $\phi$ includes the voltages and currents of the $n_{\phi}$ converters connected to the $n_{\phi}$ fast storage devices that regulate transient stability. The voltage $v_{\text {bus }}>0$ is the voltage of the bus that interconnects the microgrid devices. Then:

$$
\begin{gathered}
\eta=\operatorname{col}\left[\eta_{j}\right] \in \mathbb{R}^{3 n_{\eta}}, j \in \mathcal{I}_{\eta}, \operatorname{card}\left(\mathcal{I}_{\eta}\right)=n_{\eta} \\
\phi=\operatorname{col}\left[\phi_{l}\right] \in \mathbb{R}^{3 n_{\phi}}, l \in \mathcal{I}_{\phi}, \operatorname{card}\left(\mathcal{I}_{\phi}\right)=n_{\phi} \\
v_{\text {bus }} \in \mathbb{R}
\end{gathered}
$$

and the global state is denoted as follows:

$$
x=\left[\begin{array}{lll}
\eta^{T} & \phi^{T} & v_{\text {bus }}
\end{array}\right]^{T} \in \mathbb{R}^{3\left(n_{\eta}+n_{\phi}\right)+1} .
$$

The global model is summarized as follows, where $f, g, h$ are functions that will be defined in (9),

$$
\dot{x}=f(x)+g(x, u, d)+h(x, d)
$$

where $d$ is the exogenous input vector representing the source or load voltages and currents and $u$ is the control vector representing the duty cycles of the converters. When not explicitly specified, the state $x \in \mathbb{R}^{3\left(n_{\eta}+n_{\phi}\right)+1}$ and the exogenous input vector $d \in \mathbb{R}^{\left(n_{\eta}+n_{\phi}\right)}$ are measurable. The vector function $d$ is the aggregate vector of $\delta_{q}^{d}$ in Table II, and is assumed constant. Assumptions on considering the exogenous input vector $d$ to be constant are reasonable, as the proposed stability analysis investigates dynamics at different time-scales with respect to the ones modifying the exogenous input [22]. We assume $v_{\text {bus }}<V_{l}$ for a), and $v_{\text {bus }}>V_{l}$ for b). The system parameters in Fig. 1 and $C_{b u s}$ are known, where $C_{\text {bus }}$ represents the value of the capacitor of the DC bus. The control vector $u \in \mathbb{R}^{\left(n_{\eta}+n_{\phi}\right)}$ contains the duty cycles of the converters. The dynamical equations of the microgrid is therefore a composition of the dynamical equations of the converters and their interconnection:

$$
\begin{cases}\dot{\eta}_{j}=A_{j} \eta_{j}+B_{j} \eta_{j} u_{j}+D_{j}+G_{j} v_{b u s}, & j \in \mathcal{I}_{\eta} \\ \dot{\phi}_{l}=A_{l} \phi_{l}+B_{l} \phi_{l} u_{l}+D_{l}+G_{l} v_{b u s}, & l \in \mathcal{I}_{\phi} \\ \dot{v}_{\text {bus }}=\frac{1}{C_{b u s}}\left[\sum_{j \in \mathcal{I}_{\eta}} \frac{v_{j}^{b}-v_{b u s}}{R_{j}^{b}}+\sum_{l \in \mathcal{I}_{\phi}} \frac{v_{l}^{b}-v_{b u s}}{R_{l}^{b}}\right]\end{cases}
$$

where the involved matrices of the converters are reported in Table I and II. Unidirectional and bidirectional step-down or step-up converters are considered.

Due to the presence of both step-up and step-down converters, the stability analysis of (9) is complex. According to the control objectives introduced in Section III, we propose to decouple the dynamics of the system (9) into two to reduce complexity. One system describes the dynamics of the slow devices (loads, sources and storage devices) with respect to the DC voltage bus at the equilibrium $v_{b u s}^{e}$, and the other represents the devices' interconnection and the fast devices.

Furthermore, we consider input capacitors of the $n_{\phi}$ converters to be negligible with respect to the size of the voltage source (see $[45,46])$. For this reason, we assume

$$
v_{l}^{d}=V_{l}, \quad \phi_{l}=\left[v_{l}^{b} i_{l}\right]^{T} \in \mathbb{R}^{2}, \forall l \in \mathcal{I}_{\phi} .
$$


Consequently, the simplified decoupled model is:

$$
\begin{gathered}
\left\{\begin{array}{c}
\left.\dot{i}_{l}=-\frac{1}{L_{l}} v_{l}^{b}-\frac{R_{q}^{s}+R_{q}^{r}}{L_{l}} i_{l}+\frac{1}{L_{l}} v_{l}^{d} u_{l}, \quad l \in \mathcal{I}_{\phi}, \quad a\right) \\
\left.\dot{i}_{l}=\frac{1}{L_{l}} v_{l}^{d}-\frac{R_{q}^{s}+R_{q}^{r}}{L_{l}} i_{l}-\frac{1}{L_{l}} v_{l}^{b} u_{l}, \quad l \in \mathcal{I}_{\phi}, \quad b\right) \\
\dot{v}_{q}^{b}=-\left[\frac{g_{q}^{b}}{C_{q}^{b}}+\frac{1}{R_{q}^{b} C_{q}^{b}}\right] v_{q}^{b}+\frac{1}{R_{q}^{b} C_{q}^{b}} v_{b u s}+\frac{1}{C_{q}^{b}} i_{q}+ \\
\quad-\frac{1}{C_{q}^{b}} i_{q} u_{q} \zeta_{q}, \quad q \in \mathcal{I}_{\eta} \cup \mathcal{I}_{\phi} \\
\dot{v}_{b u s}=\frac{1}{C_{b u s}}\left[\sum_{j \in \mathcal{I}_{\eta}} \frac{v_{j}^{b}-v_{b u s}}{R_{j}^{b}}+\sum_{l \in \mathcal{I}_{\phi}} \frac{v_{l}^{b}-v_{b u s}}{R_{l}^{b}}\right]
\end{array}\right. \\
\dot{\eta}_{j}=A_{j} \eta_{j}+B_{j} \eta_{j} u_{j}+D_{j}+G_{j} v_{b u s}^{e}, \quad j \in \mathcal{I}_{\eta}
\end{gathered}
$$

where $\zeta_{q}=0$ in case the device is of type a), $\zeta_{q}=1$ otherwise. The system in (11) represents the subsystems' interconnection and the fast devices, and the one in (12) describes the dynamics of slow devices with the DC bus at steady state. We focus on DC bus voltage regulation, and therefore consider control objectives (I) and (II) reached in (12). For this reason, we suppose there exist control inputs $u_{j}, j \in \mathcal{I}_{\eta}$, such that the variables in (12) converge to the given set-points $[4,18]$. Then, we define control laws $u_{l}, l \in \mathcal{I}_{\phi}$, at the aim that system (11) admits a steady-state equilibrium point and $v_{\text {bus }}$ converges to its reference value.

This decoupling is common practice in power systems applications. Indeed, the problem of ensuring short-term voltage stability is usually treated without considering the dynamics of the devices responsible for long-term stability, i.e. the slow devices [13], [23], [22]. The decoupling can be obtained via multi-time scale analysis, as in [47]. Singular perturbation theory (see [48], [49], [27], [26]) can be used to derive controllers that decouple the dynamics with respect to the desired objective, as for example in [50]. The dynamics of the output capacitor of the converters together with the DC bus dynamics describes the subsystems interconnection, and for this reason, it is included in subsystem (11). We use the decoupled system to derive the control laws and to study stability. The results so obtained will be validated with respect to the model in (9) by simulations.

The voltage reference for $v_{\text {bus }}$ is a constant value $\bar{v}_{\text {bus }}$ determined by a design decision. The steady-state values for system (11) are the set-points given by the higher level controllers combined with the solutions of the equations in (11) and (12) at equilibrium. Let $\bar{v}_{q}^{b}, \bar{i}_{q}, \bar{u}_{q}$ be the values at steady-state of the state variables $v_{q}^{b}, i_{q}$, and of the control law $u_{q}, q \in\left\{\mathcal{I}_{\eta} \cup \mathcal{I}_{\phi}\right\}$ : the steady-state power balance condition can be expressed as

$$
\bar{\Psi}_{\mathcal{I}_{\eta}}=\sum_{j \in \mathcal{I}_{\eta}}\left[\bar{v}_{j}^{b} \bar{i}_{j}\left(1-\bar{u}_{j} \zeta_{j}\right)-g_{j}^{b}\left(\bar{v}_{j}^{b}\right)^{2}\right]=0
$$

where $\zeta_{j}=0$ in case the device is of type a), $\zeta_{j}=1$ otherwise.

A graphical representation of a star topology DC microgrid is given in Fig. 2. We assume that each subsystem knows the DC bus voltage. We consider three cases: i) the completeinformation case, where the controller of each fast device knows the complete state of the system; ii) the partialinformation case, where each controller of the fast devices
TABLE II

THE DIFFERENT PARAMETERS OF THE MATRICES ACCORDING TO THE CONSIDERED CASE: Voltage Source, CuRRent SOURCE, CIL, CPL.

\begin{tabular}{|c|c|c|c|c|}
\hline & Voltage & Current & CIL & CPL \\
\hline$\delta_{q}$ & $1 /\left(R_{q}^{d} C_{q}^{d}\right)$ & 0 & 0 & 0 \\
\hline$\delta_{q}^{d}$ & $V_{q} /\left(R_{q}^{d} C_{q}^{d}\right)$ & $I_{q} /\left(C_{q}^{d}\right)$ & $v_{q}^{d} /\left(R_{q}^{L} C_{q}^{d}\right)$ & $P_{q}^{L} / v_{q}^{d}$ \\
\hline
\end{tabular}

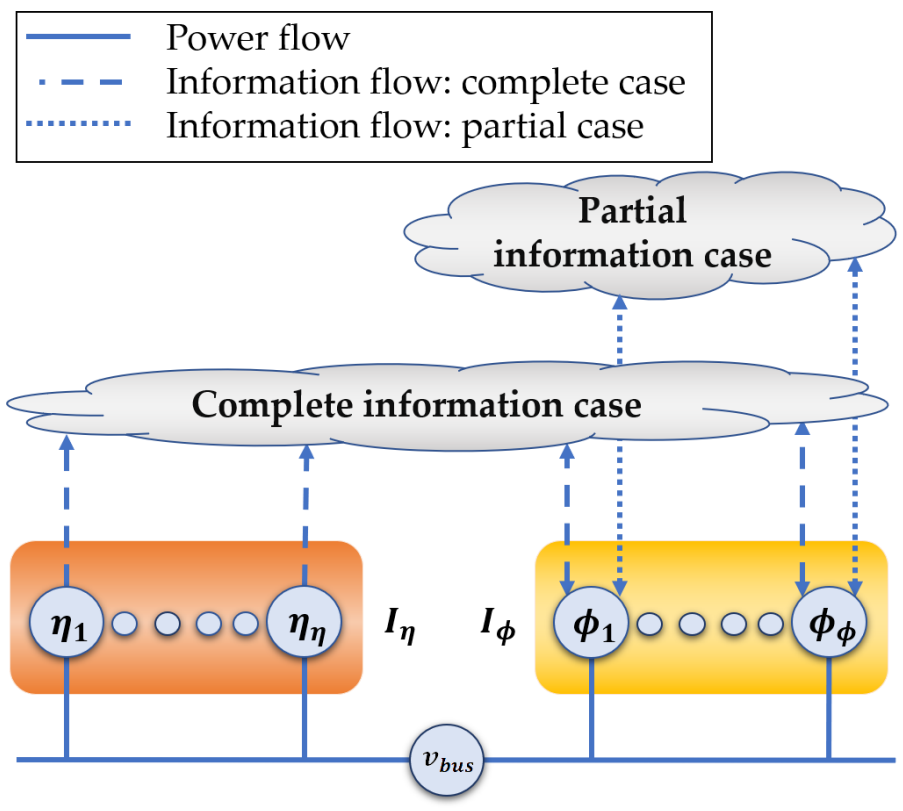

Fig. 2. The DC microgrid and its communication architecture.

knows the state of the other fast devices, but has no knowledge of the other subsystems. iii) the no-information case, where the controller of each subsystem has only information related to the variables of its subsystem.

The control problem addressed in this paper is:

Problem 1. Given the system in (11) and under the assumptions (1), (2), (3), find control laws to achieve objective (III) while ensuring the steady-state power balance (13) and voltage regulation around the reference value of $v_{b u s}$ by current sharing.

\section{DEVICE CONTROL}

In this section, we propose a systematic procedure for controlling each fast storage device connected to the DC bus. The proposed control laws are introduced with respect to a reference. Each state variable in (6) and (10) of the system in (11) will be analyzed according to its characteristics (step-up or step-down) and the proper control reference. Each subsystem is not fully linearizable by static nonlinear feedback since its maximum relative degree is two or one, according to a) or b) in Table I.

We define the error variables $\tilde{v}_{l}^{b}$ and $\tilde{i}_{l}$ depending on the voltage reference $z_{l}$ and on the current reference $r_{l}$ as

$$
\tilde{v}_{l}^{b}=v_{l}^{b}-z_{l}, \quad \tilde{i}_{l}=i_{l}-r_{l}, \quad \forall l \in \mathcal{I}_{\phi} .
$$


The control law $u_{l}, l \in \mathcal{I}_{\phi}$, depends on the current reference $r_{l}, l \in \mathcal{I}_{\phi}$, and on the voltage reference $z_{l}, l \in \mathcal{I}_{\phi}$, to be computed according to each subsystem characteristics:

$$
\begin{aligned}
& \text { a) } u_{l}=\frac{1}{v_{l}^{d}}\left[\left(R_{l}^{s}+R_{l}^{r}\right) r_{l}+z_{l}+L_{l} \dot{r}_{l}-K_{l}^{i} \tilde{i}_{l}\right], \\
& \text { b) } u_{l}=\frac{1}{v_{l}^{b}}\left[v_{l}^{d}-\left(R_{l}^{s}+R_{l}^{r}\right) r_{l}-L_{l} \dot{r}_{l}+K_{l}^{i} \tilde{i}_{l}\right],
\end{aligned}
$$

with $K_{l}^{i}>0, l \in \mathcal{I}_{\phi}$. The control laws in (15) and (16) are obtained using nonlinear control methods as backstepping, singular perturbation theory, dynamic feedback linearization and Lyapunov methods (see [26], [27]). Computation of the proposed control laws (15) and (16) are reported in Appendix $\mathrm{A}$, and the reference values used are described below.

\section{A. Reference choice for fast devices in $\mathcal{I}_{\phi}$}

These devices stabilize the DC bus by tracking a trajectory $z_{l}$ for $v_{l}^{b}$.

1) Trajectory choice for DC bus voltage regulation: The trajectory $z_{l}$ is different according to the information level. It is computed using backstepping (see [26], [27]).

- Distributed control case: complete-information of state and control variables

Let

$$
\Psi_{\mathcal{I}_{\eta}}=\sum_{j \in \mathcal{I}_{\eta}} v_{j}^{b}\left[i_{j}\left(1-u_{j} \zeta_{j}\right)-g_{j}^{b} v_{j}^{b}\right],
$$

where $\zeta_{j}=0$ in case the devices is of type a), $\zeta_{j}=1$ otherwise. Here $\Psi_{\mathcal{I}_{\eta}}$ describes a time-varying perturbation whose dynamics are generated by the (known) exosystem in (12). We choose $z_{l}, l \in \mathcal{I}_{\phi}$, as follows:

$$
z_{l}=v_{\text {bus }}-\frac{R_{l}^{b}}{v_{\text {bus }}} \gamma_{l}\left[\Psi_{\mathcal{I}_{\eta}}+K_{l}\left(v_{\text {bus }}^{2}-\bar{v}_{\text {bus }}^{2}\right)\right], \quad K_{l}>\frac{1}{R_{l}^{b}},
$$

where each gain $\gamma_{l}$ is such that

$$
\gamma_{l} \in[0,1], \quad \sum_{l \in \mathcal{I}_{\phi}} \gamma_{l}=1
$$

Therefore, $\gamma_{l} \in \Omega_{\gamma}$, where

$$
\Omega_{\gamma}=\left\{\gamma_{l} \in[0,1]: \sum_{l \in \mathcal{I}_{\phi}} \gamma_{l}=1\right\} \text {. }
$$

- Partially distributed control case: partial-information of state and control variables

Our choice of $z_{l}$ is

$$
z_{l}=v_{\text {bus }}-\frac{R_{l}^{b}}{v_{\text {bus }}} \gamma_{l}\left[K_{l}\left(v_{\text {bus }}^{2}-\bar{v}_{\text {bus }}^{2}\right)\right], \quad K_{l}>\frac{1}{R_{l}^{b}},
$$

where the gains $\gamma_{l}$ satisfy condition (19).

- Decentralized control case: no-information

The reference $z_{l}$ is

$$
z_{l}=v_{\text {bus }}-\frac{R_{l}^{b}}{v_{\text {bus }}}\left[K_{l}\left(v_{\text {bus }}^{2}-\bar{v}_{b u s}^{2}\right)\right], \quad K_{l}>\frac{1}{R_{l}^{b}} .
$$

2) Reference current trajectories: Because the relative degree of the converter is different according to its topology, $r_{l}$ is computed by either 1) using backstepping (see [26], [27]) or 2) using backstepping, dynamic feedback linearization and singular perturbation theory (see [29], [42], [48]).

1) Step-down case, a) in Table $I$.

The choice of $r_{l}$ is

$$
r_{l}=\left(g_{l}^{b}+\frac{1}{R_{l}^{b}}\right) z_{l}-\frac{1}{R_{l}^{b}} v_{b u s}+C_{l}^{b} \dot{z}_{l}-K_{l}^{b} \tilde{v}_{l}^{b} .
$$

\section{2) Step-up case (b) in Table I).}

Based on singular perturbation theory and similarly to [42], we apply control law (16) in steady-state to $v_{l}^{b}$ in (11). To obtain $r_{l}$, we use dynamic feedback linearization (see [29], [28]). Using a Lyapunov-based approach, we consider the function $\dot{f}_{l}^{b}$ that includes the term $\dot{\tilde{v}}_{l}^{b}$. We differentiate $\dot{f}_{l}^{b}$, to obtain a function $\ddot{f}_{l}^{b}$ :

$$
\begin{aligned}
\dot{f}_{l}^{b} & =\frac{1}{R_{l}} v_{\text {bus }}-\left(g_{l}^{b}+\frac{1}{R_{l}}\right) z_{l}+r_{l}-C_{l} \dot{z}_{l} \\
& -\frac{1}{\tilde{v}_{l}^{v}+z_{l}} r_{l}\left(v^{d}+\left(R^{s}+R^{r}\right) r_{l}\right)=L_{f_{v}}^{1}, \\
\ddot{f}_{l}^{b} & =\frac{1}{R_{l}} \dot{v}_{b u s}-\left(g_{l}^{b}+\frac{1}{R_{l}}\right) \dot{z}_{l}-C_{l} \ddot{z}_{l} \\
& -\frac{1}{\left(\tilde{v}_{l}^{v}+z_{l}\right)^{2}} r_{l}\left(\dot{\tilde{v}}_{l}^{v}+\dot{z}_{l}\right)\left(v^{d}+\left(R^{s}+R^{r}\right) r_{l}\right) \\
+ & \dot{r}_{l}\left[1-\frac{v^{d}+2\left(R^{s}+R^{r}\right) r_{l}}{\tilde{v}_{l}^{v}+z_{l}}\right] .
\end{aligned}
$$

Then $\ddot{f}_{l}^{b}=L_{f_{v}}^{2}+L_{g_{v}} \dot{r}_{l}$, where terms $L_{f_{v}}^{2}$ and $L_{g_{v}}$ (notation of Lie derivatives is used) are obtained from (25).

Considering $\dot{r}_{l}$ as a virtual input, $r_{l}$ is obtained by integrating $\dot{r}_{l}$ :

$$
r_{l}(t)=\int_{0}^{t} \frac{1}{L_{g_{v}}}\left[-L_{f_{v}}^{2}-\tilde{v}_{l}^{b}-\bar{K}_{f} \dot{f}_{v}\right] d \tau
$$

with $\bar{K}_{f}>0$. Details on the choice of $r_{l}$ are given in Appendix A.

Let the state vector $\tilde{\phi}_{l}$ be:

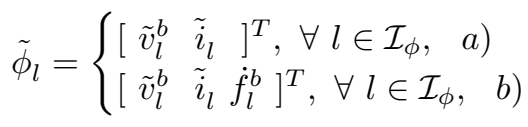

\section{LYAPUNOV FUNCTIONS}

In this section we leverage results available in the literature, and in particular, [4], [26], [27], [29], [42], [51], to analyse the stability of each device. We will derive Lyapunov functions for each subsystem.

\section{A. Lyapunov functions for the interconnection subsystem}

In order to deal with system interconnection stability, we define the following Lyapunov function $W^{\text {bus }}\left(v_{j}^{b}, v_{b u s}\right)$ :

$$
W^{\text {bus }}\left(v_{j}^{b}, v_{\text {bus }}\right)=\sum_{j \in \mathcal{I}_{\eta}} \frac{C_{j}^{b}}{2}\left(v_{j}^{b}\right)^{2}+\frac{C_{b u s}}{2} v_{b u s}^{2}
$$


that represents the energy stored in the DC bus and in the output capacitors of the DC/DC converters connected to the slow devices. The three cases of information sharing among the devices are investigated: complete-information, partialinformation and no-information. They result in three control approaches: distributed control, partially distributed control and decentralized control, respectively. Figure 2 shows the complete and partial information sharing cases.

The time derivative of $W^{\text {bus }}$ in (28) is

$$
\dot{W}^{\text {bus }}=-\Psi_{\mathcal{I}_{\eta}, v_{\text {bus }}}+v_{\text {bus }} \Psi_{\mathcal{I}_{\phi}, v_{b u s}}+\Psi_{\mathcal{I}_{\eta}}
$$

where $\Psi_{\mathcal{I}_{\eta}}$ is defined in (17), and

$\Psi_{\mathcal{I}_{\eta}, v_{\text {bus }}}=\sum_{j \in \mathcal{I}_{\eta}} \frac{\left(v_{j}^{b}-v_{\text {bus }}\right)^{2}}{R_{j}^{b}}, \quad \Psi_{\mathcal{I}_{\phi}, v_{\text {bus }}}=\sum_{l \in \mathcal{I}_{\phi}} \frac{\left(v_{l}^{b}-v_{\text {bus }}\right)}{R_{l}^{b}}$.

1) Distributed control case: complete-information of state and control variables: Each controller of the subsystems in $\mathcal{I}_{\phi}$ has information about the state and control inputs of the other subsystems, i.e., $\Psi_{\mathcal{I}_{\eta}}$ is known to each $u_{l}$, and can be used in the calculation of $z_{l}$. Each $z_{l}, l \in \mathcal{I}_{\phi}$, is chosen as in (18) such that condition (19) is satisfied.

Similarly to the droop gains, the gains $\gamma_{l}$ that satisfy condition (19) allow for the sharing of the current needed to regulate the DC bus voltage. The resulting $\dot{W}^{\text {bus }}$ is:

$$
\begin{aligned}
\dot{W}^{\text {bus }}= & -\Psi_{\mathcal{I}_{\eta}, v_{\text {bus }}}-\sum_{l \in \mathcal{I}_{\phi}}\left(\frac{1}{R_{l}^{b}} \gamma_{l} K_{l}\right)\left(v_{\text {bus }}^{2}-\bar{v}_{\text {bus }}^{2}\right) \\
& +v_{\text {bus }} \sum_{l \in \mathcal{I}_{\phi}} \frac{1}{R_{l}^{b}} \tilde{v}_{l}^{b} .
\end{aligned}
$$

2) Partially distributed control case:

partial-information of state and control variables: The term $\Psi_{\mathcal{I}_{\eta}}$ in (29) is unknown. However, from Assumptions 1 and 3 , it can be modeled as an unknown bounded disturbance. Because of the communications taking place among the fast devices, they can still balance their control contribution through $\gamma_{l}$, similarly to (18). From Assumption 3 and the exogenous input vector being constant, the boundaries of $\Psi_{\mathcal{I}_{\eta}}$ are known. $\Psi_{\mathcal{I}_{\eta}}$ is now seen as a disturbance, and $\left|\Psi_{\mathcal{I}_{\eta}}\right|,\left|\dot{\Psi}_{\mathcal{I}_{\eta}}\right| \leq \Psi_{\max }$. The $\dot{W}^{\text {bus }}$ in (29) is then rewritten as

$$
\begin{aligned}
\dot{W}^{\text {bus }} & =-\Psi_{\mathcal{I}_{\eta}, v_{b u s}}+v_{\text {bus }} \Psi_{\mathcal{I}_{\phi}, v_{b u s}}+\Psi_{\mathcal{I}_{\eta}} \\
& \leq-\Psi_{\mathcal{I}_{\eta}, v_{b u s}}+v_{b u s} \Psi_{\mathcal{I}_{\phi}, v_{b u s}}+\Psi_{\text {max }}
\end{aligned}
$$

The choice of $z_{l}$ in (21) where the gains $\gamma_{l}$ respect condition (19), ensures the possibility to share the control effort. Equation (32) can be rewritten as

$$
\begin{aligned}
\dot{W}^{\text {bus }} & =-\Psi_{\mathcal{I}_{\eta}, v_{\text {bus }}}-\sum_{l \in \mathcal{I}_{\phi}}\left(\frac{1}{R_{l}^{b}}+\gamma_{l} K_{l}\right)\left(v_{\text {bus }}^{2}-\bar{v}_{\text {bus }}^{2}\right)+ \\
& +\Psi_{\max }+v_{\text {bus }} \sum_{l \in \mathcal{I}_{\phi}} \frac{1}{R_{l}^{b}} \tilde{v}_{l}^{b}
\end{aligned}
$$

3) Decentralized control case: no-information: In this case, the term $\Psi_{\mathcal{I}_{\eta}}$ in (29) continues to be seen as a disturbance, but each device is supposed to deal with it in a noncooperative mode, without the possibility to share the needed power. The resulting $\dot{W}^{\text {bus }}$ is as (32), and the reference $z_{l}$ is as (22). Then, we have a result similar to (33). However, here, it is not possible to choose the amount of power provided by each device, and consequently no current sharing is achievable.

\section{B. Lyapunov functions for fast devices}

The Lyapunov function $W^{\phi}\left(\tilde{\phi}_{l}\right)$ used to verify stability is:

$$
\begin{aligned}
W^{\phi}\left(\tilde{\phi}_{l}\right)= & W_{\mathcal{I}_{\phi}}^{i}\left(\tilde{i}_{l}\right)+W_{\mathcal{I}_{\phi}}^{b}\left(\tilde{v}_{l}^{b}, \dot{f}_{l}^{b}\right) \zeta_{l} \\
& +W_{\mathcal{I}_{\phi}}^{b}\left(\tilde{v}_{l}^{b}\right)\left(1-\zeta_{l}\right), \quad \forall l \in \mathcal{I}_{\phi},
\end{aligned}
$$

where $\zeta_{l}=0$ in case the device is of type a), $\zeta_{q}=1$ otherwise. $W_{\mathcal{I}_{\phi}}^{i}$ is defined as

$$
W_{\mathcal{I}_{\phi}}^{i}=\sum_{l \in \mathcal{I}_{\phi}} \frac{L_{l}}{2}\left(\tilde{i}_{l}\right)^{2}
$$

and $W_{\mathcal{I}_{\phi}}^{b}$ is defined in accordance to a) or b).

Selecting the references in (23) and (26), we have:

1) Step-down case (a) in Table I).

$W_{\mathcal{I}_{\phi}}^{b}$ is defined as

$$
W_{\mathcal{I}_{\phi}}^{b}=\sum_{l \in \mathcal{I}_{\phi}} \frac{C_{l}^{b}}{2}\left(\tilde{v}_{l}^{b}\right)^{2}
$$

Given $u_{l}$ in (15) with respect to the references $r_{l}$ in (23), there exists matrix $Q_{l}^{A}>0$ such that the time derivatives of $W_{\mathcal{I}_{\phi}}^{i}$ and $W_{l}^{b}$ defined in (35) and (36) with respect to $W^{\phi}$ in (34) are negative definite, i.e.

$$
\begin{aligned}
\dot{W}^{\phi}= & -\sum_{l \in \mathcal{I}_{\phi}}\left[\tilde{v}_{l}^{b} \tilde{i}_{l}\right] \underbrace{\left[\begin{array}{rr}
g_{l}^{b}+\frac{1}{R_{l}^{b}}+K_{l}^{b} & 0 \\
0 & R_{l}^{s}+R_{l}^{r}+K_{l}^{i}
\end{array}\right]}_{Q_{l}^{A}}\left[\begin{array}{c}
\tilde{v}_{l}^{b} \\
\tilde{i}_{l}
\end{array}\right] \\
& -v_{\text {bus }} \sum_{l \in \mathcal{I}_{\phi}} \frac{1}{R_{l}^{b}} \tilde{v}_{l}^{b}
\end{aligned}
$$

Stability results are computed applying the backstepping technique to $\dot{W}^{\text {bus }}$ (see $[26,27]$ ). The reader is referred to Appendix A1 for further details.

2) Step-up case (b) in Table I).

We consider a function $W_{\mathcal{I}_{\phi}}^{i}$ in the form of (35) to find the control $u_{l}$ in (16) ensuring convergence of $i_{l}$ to $r_{l}$. Then, $W_{\mathcal{I}_{\phi}}^{b}$ is

$$
W_{\mathcal{I}_{\phi}}^{b}=\sum_{l \in \mathcal{I}_{\phi}}\left[\frac{C_{l}^{b}}{2}\left(\tilde{v}_{l}^{b}\right)^{2}+\frac{1}{2}\left(\dot{f}_{l}^{b}\right)^{2}\right] .
$$

In accordance to (25) and $r_{l}$ in (26),

$$
\begin{aligned}
& \dot{W}^{\phi}=-v_{\text {bus }} \sum_{l \in \mathcal{I}_{\phi}} \frac{1}{R_{l}^{b}} \tilde{v}_{l}^{b} \\
& -\sum_{l \in \mathcal{I}_{\phi}}\left[\begin{array}{lll}
\tilde{v}_{l}^{b} & \dot{f}_{l}^{b} & \tilde{i}_{l}
\end{array}\right] \underbrace{\left[\begin{array}{ccc}
g_{l}^{b}+\frac{1}{R_{l}^{b}} & 0 & 0 \\
0 & \bar{K}_{f} & 0 \\
0 & 0 & R_{l}^{s}+R_{l}^{r}+K_{l}^{i}
\end{array}\right]}_{Q_{l}^{B}}\left[\begin{array}{c}
\tilde{v}_{l}^{b} \\
\dot{f}_{l}^{b} \\
\tilde{i}_{l}
\end{array}\right] .
\end{aligned}
$$

These functions are obtained by applying backstepping and dynamic feedback linearization techniques together with singular perturbation theory (see [26], [27], [29]) (See Appendix A2 for further details). 


\section{SySTEM STABILITY ANALYSIS}

In this section, we combine the functions of the previous section to assess ISS for the entire system. We will show that the control laws solve Problem 1 under all information scenarios. ISS is of interest in power systems, and in particular, in microgrids, where each control level depends on external set-points fixed by higher level controllers and external disturbances act on the system [7]. In a microgrid, some state variables represent electrical power generators while others represent loads, storage systems and connection lines. Our analysis based on the composition of Lyapunov functions to prove ISS allows separating the different characteristics of the devices.

Let us consider the extended state $\Upsilon$ of the subsystems in (11) and the variables added for control purposes $\dot{f}_{l}^{b}$ in $\tilde{\phi}_{l}$ in (27):

$$
\Upsilon(t)=\left[\operatorname{col}\left[\tilde{\phi}_{l}, l \in \mathcal{I}_{\phi}\right] ; \quad \operatorname{col}\left[v_{j}^{b}, j \in \mathcal{I}_{\eta}\right] ; v_{\text {bus }}\right] .
$$

We consider a set $\Omega_{d}$ and a set $\Omega_{\Upsilon}$ such that for any $\Upsilon(0) \in$ $\Omega_{\Upsilon}$ and for any $d \in \Omega_{d}$ the input function $u_{l}$ in (15) and (16) is well defined (finite), and $\Upsilon(t) \in \Omega_{\Upsilon}, \forall t \geq 0$. The variables $v_{q}^{b}$ and $v_{b u s}$ are always strictly positive because of the physics of the system. Thus, the control laws in (15) and (16) are always well defined. For physical reasons, we choose the set $\Omega_{\Upsilon}$ to be

$$
\Omega_{\Upsilon}=\left\{\Upsilon \in \mathbb{R}^{2 n_{\phi}+n_{\eta}+1}: v_{q}^{b}, v_{\text {bus }}>0, q \in\left\{\mathcal{I}_{\phi} \cup \mathcal{I}_{\eta}\right\}\right\} .
$$

We assume that the set $\Omega_{\gamma}$ of $\gamma_{l}$ respecting condition (19) and the set-points from the higher level controller are given.

\section{A. Distributed case}

Theorem 1. In the distributed control case, given:

1) the set $\Omega_{\gamma}$;

2) the exogenous input $d$;

3) the working set-points;

4) the voltage reference $\bar{v}_{\text {bus }}$;

5) the reference $z_{l}, l \in \mathcal{I}_{\phi}$, in (18).

Let the solution of the closed loop system (11), (15), (23) (case a) and (11), (16), (26) (case b) exist and stay in $\Omega_{\Upsilon}$ (see (41)) $\forall t \in \mathbb{R}^{+}$.

Then, there exist functions $\beta \in \mathcal{K} \mathcal{L}$ and $\omega \in \mathcal{K}$ such that the following inequality holds:

$$
|\Upsilon(t)| \leq \beta(\Upsilon(0), t)+\omega\left(\bar{v}_{\text {bus }}\right), \forall \Upsilon(0) \in \Omega_{\Upsilon}, \forall t \in \mathbb{R}^{+} .
$$

Proof. Let $W$ be the Lyapunov function composed of the Lyapunov functions introduced in the previous section. Using (28), (34), (35), (36), (38), $W$ is

$$
\begin{aligned}
W(\Upsilon(t))= & W^{\phi}+W^{\text {bus }} \\
= & \sum_{l \in \mathcal{I}_{\phi}} \frac{L_{l}}{2}\left(\tilde{i}_{l}\right)^{2}+\sum_{l \in \mathcal{I}_{\phi}}\left(1-\zeta_{l}\right) \frac{C_{l}^{b}}{2}\left(\tilde{v}_{l}^{b}\right)^{2} \\
& +\sum_{l \in \mathcal{I}_{\phi}} \zeta_{l}\left[\frac{C_{l}^{b}}{2}\left(\tilde{v}_{l}^{b}\right)^{2}+\frac{1}{2}\left(\dot{f}_{l}^{b}\right)^{2}\right] \\
& +\sum_{j \in \mathcal{I}_{\eta}} \frac{C_{j}^{b}}{2}\left(v_{j}^{b}\right)^{2}+\frac{C_{b u s}}{2} v_{\text {bus }}^{2},
\end{aligned}
$$

where $\zeta_{l}=0$ in case the device is of type a), $\zeta_{q}=1$ otherwise. Consequently, from the quadratic structure of $W$ in (43), there exist functions $\underline{\alpha}, \bar{\alpha} \in \mathcal{K}_{\infty}$ such that

$$
\underline{\alpha}(|\Upsilon(t)|) \leq W(\Upsilon(t)) \leq \bar{\alpha}(|\Upsilon(t)|), t \geq 0 .
$$

We compute the time derivative of $W(\Upsilon(t))$ in (43) according to (29), (34), (37) and (39), as

$$
\begin{aligned}
& \dot{W}(\Upsilon(t))=\dot{W}^{\phi}+\dot{W}^{\text {bus }} \\
& \leq-\sum_{l \in \mathcal{I}_{\phi}}\left(1-\zeta_{l}\right)\left[\begin{array}{ll}
\tilde{v}_{l}^{b} & \tilde{i}_{l}
\end{array}\right] Q_{l}^{A}\left[\begin{array}{c}
\tilde{v}_{l}^{b} \\
\tilde{i}_{l}
\end{array}\right] \\
& -\sum_{l \in \mathcal{I}_{\phi}} \zeta_{l}\left[\begin{array}{lll}
\tilde{v}_{l}^{b} & \dot{f}_{l}^{b} & \tilde{i}_{l}
\end{array}\right] Q_{l}^{B}\left[\begin{array}{c}
\tilde{v}_{l}^{b} \\
\dot{f}_{l}^{b} \\
\tilde{i}_{l}
\end{array}\right] \\
& \underbrace{-\sum_{j \in \mathcal{I}_{\eta}} \frac{\left(v_{j}^{b}-v_{b u s}\right)^{2}}{R_{j}^{b}}-\sum_{l \in \mathcal{I}_{\phi}} \gamma_{l} K_{l} v_{b u s}^{2}}_{\Psi}+\sum_{l \in \mathcal{I}_{\phi}} \gamma_{l} K_{l}\left(\bar{v}_{b u s}\right)^{2} .
\end{aligned}
$$

The Lyapunov function $W^{\text {bus }}$ in (28) and (43) is equal to zero at zero, while its time derivative in (31) and (45) holds only in $\Omega_{\Upsilon}$. However, the function $\Psi$ in (45) is a negative definite quadratic function of the variables $\left[\operatorname{col}\left[v_{j}^{b}, j \in\right.\right.$ $\left.\left.\mathcal{I}_{\eta}\right] ; v_{\text {bus }}\right]$. Then, from the quadratic structure of the time derivative in (45) and from the considerations on $\Psi$, there exist functions $\beta_{\Upsilon}, \gamma_{\Upsilon} \in \mathcal{K}_{\infty}$ such that the following inequality holds:

$$
\dot{W}(\Upsilon(t)) \leq-\beta_{\Upsilon}(|\Upsilon(t)|)+\gamma_{\Upsilon}\left(\left|\bar{v}_{b u s}\right|\right) .
$$

From (44) and (46), by Lemma 2.14, pp. 82-84, in [52], inequality (42) follows.

The control laws $u_{l}$ in (15) and (16), the references $r_{l}$ in (23) and (26), and the references $z_{l}$ in (18), $l \in \mathcal{I}_{\phi}$, are developed based on power flow considerations. They target steady state by matching power balance among the devices in the microgrid, thus counteracting the voltage variations and keeping the evolution of $\Upsilon(t)$ in $\Omega_{\Upsilon}$.

The Lyapunov function in (43) is similar to an ISS Lyapunov function, as described in [31], [32], [38]. The proposed result provides a typical ISS inequality for the closed loop system (11), (15), (23) (case a) and (11), (16), (26) (case b). Here $\bar{v}_{\text {bus }}$ plays the role of the virtual input, and notice that it must be positive.

\section{B. Partially distributed case}

Theorem 2. In the partially distributed control case, given:

1) the set $\Omega_{\gamma}$;

2) the exogenous input $d$;

3) the working set-points;

4) the voltage reference $\bar{v}_{b u s}$;

5) the reference $z_{l}, l \in \mathcal{I}_{\phi}$, in (21).

Let the solution of the closed loop system (11), (15), (23) (case a) and (11), (16), (26) (case b) exist and stay in $\Omega_{\Upsilon}$ (see (41)) $\forall t \in \mathbb{R}^{+}$. 
Then, there exist functions $\beta \in \mathcal{K} \mathcal{L}, \omega \in \mathcal{K}$ and a positive real $\varepsilon$ such that the following inequality holds:

$$
|\Upsilon(t)| \leq \beta(\Upsilon(0), t)+\omega\left(\bar{v}_{\text {bus }}\right)+\varepsilon \forall \Upsilon(0) \in \Omega_{\Upsilon} \forall t \in \mathbb{R}^{+} \text {. }
$$

Proof. As for Theorem 1, the proof is based on the construction of a Lyapunov function $W$ as (43) for the entire system. The difference with respect to Theorem 1 is $\dot{W}^{\text {bus }}$. Indeed, its time derivative is described by equation (32), where $\Psi_{\mathcal{I}_{\eta}}$ is unknown but bounded by $\Psi_{\max }$. Then,

$$
\begin{aligned}
\dot{W} & =-\sum_{l \in \mathcal{I}_{\phi}}\left(1-\zeta_{l}\right)\left[\begin{array}{lll}
\tilde{v}_{l}^{b} & \tilde{i}_{l}
\end{array}\right] Q_{l}^{A}\left[\begin{array}{c}
\tilde{v}_{l}^{b} \\
\tilde{i}_{l}
\end{array}\right] \\
& -\sum_{l \in \mathcal{I}_{\phi}} \zeta_{l}\left[\begin{array}{lll}
\tilde{v}_{l}^{b} & \dot{f}_{l}^{b} & \tilde{i}_{l}
\end{array}\right] Q_{l}^{B}\left[\begin{array}{c}
\tilde{v}_{l}^{b} \\
\dot{f}_{l}^{b} \\
\tilde{i}_{l}
\end{array}\right] \\
& -\sum_{j \in \mathcal{I}_{\eta}} \frac{\left(v_{j}^{b}-v_{b u s}\right)^{2}}{R_{j}^{b}}-\sum_{l \in \mathcal{I}_{\phi}} \gamma_{l} K_{l} v_{b u s}^{2} \\
& +\sum_{l \in \mathcal{I}_{\phi}} \gamma_{l} K_{l} \bar{v}_{b u s}^{2}+\Psi_{\max } .
\end{aligned}
$$

Then

$$
\dot{W}\left(\Upsilon(t), \bar{v}_{b u s}\right) \leq-\beta_{\Upsilon}(|\Upsilon(t)|)+\gamma_{\Upsilon}\left(\left|\bar{v}_{b u s}\right|\right)+\Psi_{\max }
$$

with functions $\beta_{\Upsilon}$ and $\gamma_{\Upsilon}$ defined as in (46). From (44) and (49), the condition in (47) follows, with $\varepsilon$ depending on $\Psi_{\max }$.

When we have no global knowledge of the subsystem in (11), a number of solutions that depend on the number of variables involved in the compensation of $\varepsilon$ exist. Depending on the system initial conditions and on its evolution, the set of possible solutions can be defined according to the set $\Omega_{\varepsilon}$, where

$$
\Omega_{\varepsilon}=\left\{\Upsilon:|\Upsilon| \leq \beta(\Upsilon(0), 0)+\omega\left(\bar{v}_{\text {bus }}\right)+\varepsilon\right\} .
$$

Then, due to the incomplete knowledge of the system, the interaction among $m$ state variables leads to $\infty^{m}$ possible solutions, i.e., we can obtain power balance with an infinite number of possible configurations of power supply.

The Lyapunov function in (47) is similar to a practical Input-to-State Stability Lyapunov function (see [27], [43]). The proposed result provides a typical practical ISS inequality for the closed loop system (11), (15), (23) (case a) and (11), (16), (26) (case b). If Assumption 2 is valid, the function $\Psi_{\mathcal{I}_{\eta}}$ is a vanishing perturbation function, since condition (13) implies $\Psi_{\mathcal{I}_{\eta}}=0$ at steady-state, which, in turn, implies that the proposed result reduces to be similar to ISS.

\section{Decentralized case}

Theorem 3. In the decentralized control case, given:

1) the set $\Omega_{\gamma}$;

2) the exogenous input $d$;

3) the working set-points;

4) the voltage reference $\bar{v}_{b u s}$;

5) the reference $z_{l}, l \in \mathcal{I}_{\phi}$, in (22).
Let the solution of the closed loop system (11), (15), (23) (case a) and (11), (16), (26) (case b) exist and stay in $\Omega_{\Upsilon}$ (see (41)) $\forall t \in \mathbb{R}^{+}$.

Then, there exist functions $\beta \in \mathcal{K} \mathcal{L}, \omega \in \mathcal{K}$ and a positive real $\varepsilon$ such that the following inequality holds:

$$
|\Upsilon(t)| \leq \beta(\Upsilon(0), t)+\omega\left(\bar{v}_{\text {bus }}\right)+\varepsilon \forall \Upsilon(0) \in \Omega_{\Upsilon} \forall t \in \mathbb{R}^{+} .
$$

Proof. The proof follows directly from the one for Theorem 2 in the special case $\gamma_{l}=1, \forall l \in \mathcal{I}_{\phi}$. The same considerations apply for the possible solutions.

Here the missing information reduces the effectiveness of the proposed control action. Theorem 3 yields the same result of Theorem 2 when the control inputs dedicated to interconnection stability do not share information about their state. The loss in coordination does not modify the stability results.

In the partially distributed or decentralised case, the modeling of the system interconnection with bounded disturbances may lead to solutions where the fast devices conflict for power outputs. However, voltage stability is still ensured. Since infinite solutions leading to power balance exist, it is possible that the fast devices see each other as disturbances acting on the system. A situation where some of them provide power while others take power (in general, each with different power levels) may appear. Albeit this situation does not impact stability (and is therefore not be considered in this paper), control at a higher level may be needed. This will be the subject of further analysis in a forthcoming paper.

\section{Remarks}

The model in (12) is based on the hypothesis that the DC voltage is stable. This hypothesis is verified by Theorems 1,2 and 3 with respect to (11). Here, ISS inequalities demonstrates that the fast devices counteract the voltage variations induced by the slow devices into the system interconnection, thus stabilizing the system. Note that the equilibrium points of $v_{j}^{b}, j \in \mathcal{I}_{\eta}$, are actually different from 0 , contrary to what could be inferred from (28). Here, the zero states are used only for energy considerations in ISS where power balance is ensured (see [53] for further details). Control laws $u_{j}$ similar to the ones in (15) and (16) are considered for reaching targets (I) and (II) with respect to given set-points (see [4, 18] for more details). They are used to simulate the closed loop system (12).

The stability results presented here are valid for a generic DC microgrid consisting of a number $n_{\eta}>0$ and $n_{\phi}>0$ of elements connected by step-up or step-down DC/DC converters and a single DC bus connection. They allow for the possibility to customize the DC microgrid according to specific necessities. The inclusion of new elements can be performed at design time or at implementation time. The elements of the microgrid can be inserted in Plug\&Play mode (see [17]).

\section{Simulations}

To show that the introduced control strategies are effective, we use the Matlab\&Simulink SimPowerSystems tool. Based 


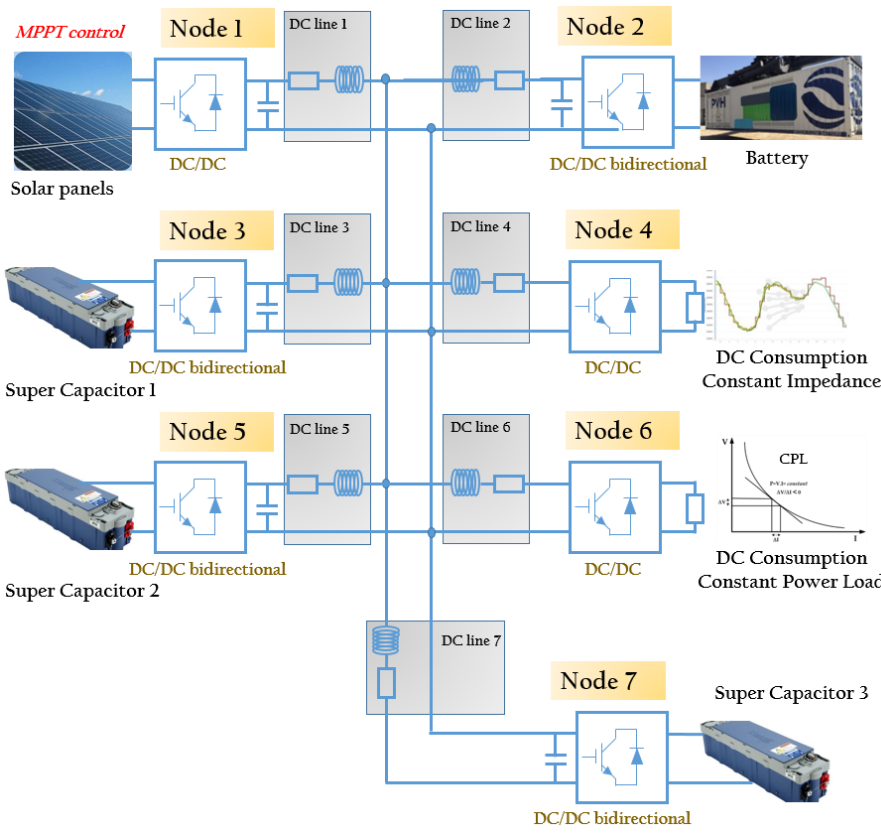

Fig. 3. The DC microgrid considered for the simulations is composed by seven nodes, with a PV source (node 1), a battery (node 2), a CIL (node 4) and a CPL (node 5), and three supercapacitors (nodes 3, 5, 7).

on the modeling in (9), a seven nodes DC microgrid is analysed, composed by PV generation, a slow storage device, three fast storage devices, a constant impedance load (CIL) and a constant power load (CPL), as shown in Figure 3. The parameters of the grid are introduced in Table III. Even if the standard QSL approximation is used to develop the controllers, inductances are considered in simulations to provide a more realistic framework.

We carried out experiments with simulation time of 15 seconds and reference changes. The PV production is variable in node 1, as shown in Fig. 4. The battery reference for the absorbed/supplied energy in node 2 varies according to the set-point given from a higher level controller (see Fig. 5). In node 4 , the constant impedance load at $\mathrm{t}=0 \mathrm{~s}$ is equal to 100 $\Omega$, and is equal to $50 \Omega$ at $\mathrm{t}=10 \mathrm{~s}$ (see Fig. 7). In node 6 , the constant power load is varying each 4 seconds, as shown in Fig. 8. In nodes 3,5 and 7 three supercapacitors of different sizes are considered. They implement the proposed current sharing control laws with respect to the considered communication framework: distributed full information, distributed partial information and decentralized case. Two different sets of values are considered for the corresponding set $\Omega_{\gamma}$. In the first case, we set $\left(\gamma_{3}=\gamma_{5}=\gamma_{7}\right)$, and in the second one we set $\gamma_{3} \neq \gamma_{5}=\gamma_{7}$. The voltage reference value for the DC bus is $160 \mathrm{~V}$. The output capacitor of the DC/DC converter connected to the battery is here taken as DC bus. Supercapacitor 1 in node 3 has a nominal voltage value of $140 \mathrm{~V}$. It is connected to the DC bus through a bidirectional step-up converter. Supercapacitors 2 and 3 (at node 5 and 7 , respectively) have nominal value of $190 \mathrm{~V}$, and are connected to the DC bus through step-down converters.

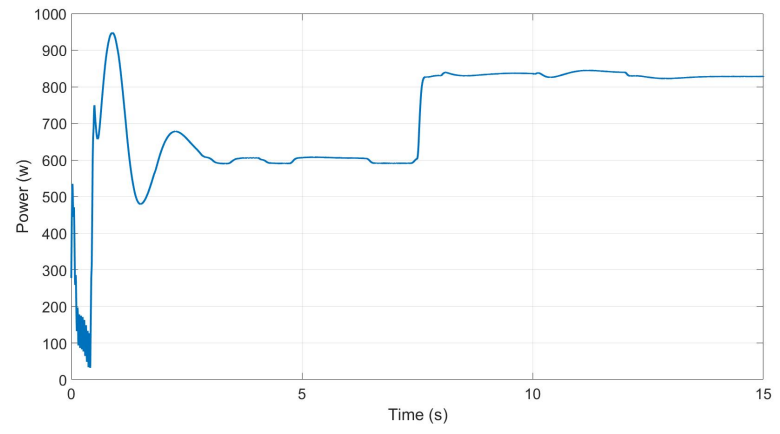

Fig. 4. The power output of the PV array in both Cases I and II.

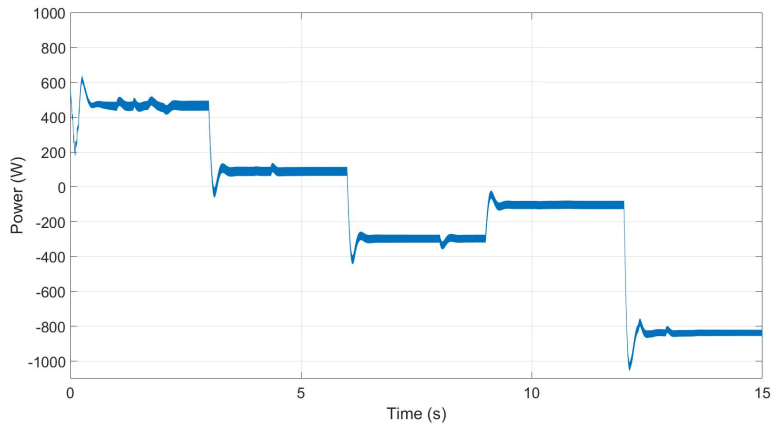

Fig. 5. The power output of the battery in both Cases I and II: the battery is supplying energy when power output is positive, while absorbing energy when negative.

\section{A. Case I: equal values for $\gamma_{l}$}

In this case, all the fast devices (supercapacitors) have the same gains: $\gamma_{3}=\gamma_{5}=\gamma_{7}=1 / 3$. Consequently, they will contribute equally in the full and partial information scenarios.

The DC voltages for the full, partial and decentralized information case are shown in Figure 6. The system response to the different kinds of disturbances is satisfactory under all three scenarios. Indeed, only a high peak is produced due to system initialization, which is under an admissible tolerance of $10 \%$. The voltage level is kept with an acceptable error, which is less than $5 \%$ with respect to the reference value $(160 \mathrm{~V})$. The control action acts faster when full information is available.

TABLE III

THE SIMULATION PARAMETERS

\begin{tabular}{|l|c|c|c|c|c|}
\hline & $\mathrm{R}$ & $\mathrm{L}$ & & & \\
\hline Line 1 & $0.5 \Omega$ & $0.1 \mathrm{H}$ & Grid & Voltage & $160 \mathrm{~V}$ \\
\hline Line 2 & $0.1 \Omega$ & $0.01 \mathrm{H}$ & PV & Voltage & $150 \mathrm{~V}$ \\
\hline Line 3 & $0.2 \Omega$ & $0.02 \mathrm{H}$ & & Power & $1 \mathrm{~kW}$ \\
\hline Line 4 & $0.5 \Omega$ & $0.01 \mathrm{H}$ & Cap1 & Capacity & $4.5 \mathrm{kWh}$ \\
\hline Line 5 & $0.3 \Omega$ & $0.03 \mathrm{H}$ & & Voltage & $140 \mathrm{~V}$ \\
\hline Line 6 & $0.1 \Omega$ & $0.01 \mathrm{H}$ & Cap2 & Capacity & $2.25 \mathrm{kWh}$ \\
\hline Line 7 & $0.15 \Omega$ & $0.02 \mathrm{H}$ & & Voltage & $190 \mathrm{~V}$ \\
\hline Battery & Capacity & $6.5 \mathrm{kWh}$ & Cap3 & Capacity & $2.25 \mathrm{kWh}$ \\
\hline & Voltage & $130 \mathrm{~V}$ & & Voltage & $190 \mathrm{~V}$ \\
\hline
\end{tabular}




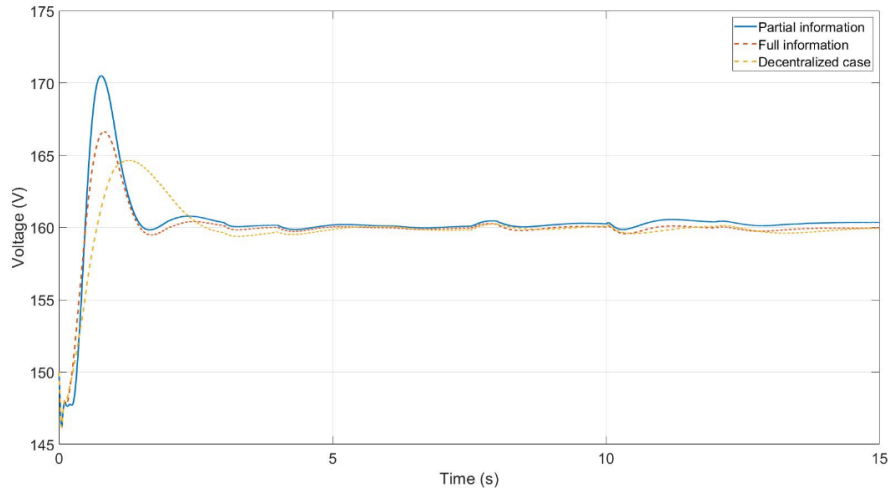

Fig. 6. Case I: The DC Microgrid voltage according to the different information sharing situations: full information (dotted red line), partial information (blue line) and no information sharing (dotted yellow line).

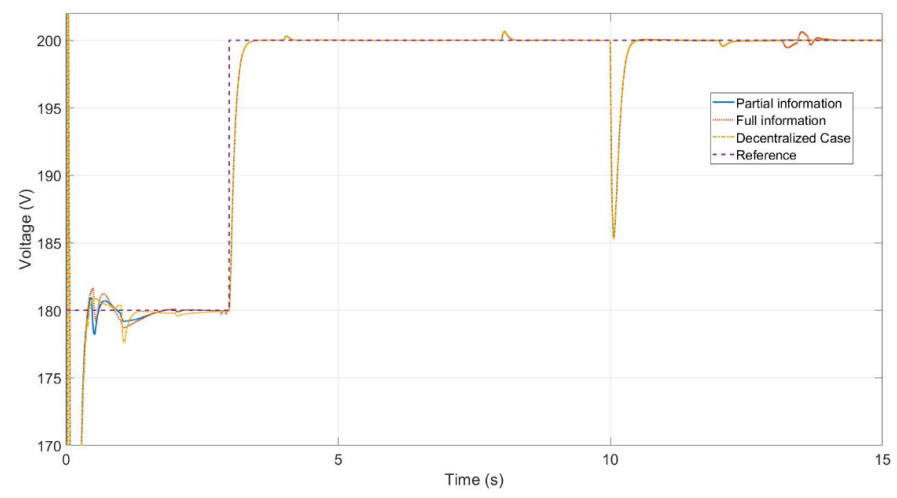

Fig. 7. Case I: The voltage in the CIL with respect to the reference (dotted violet line) in case of partial information (blue line), full information (dotted red line) and no information sharing (dotted yellow line).

However, when full information spreading is not available, control action still performs well under the load variations shown in Figures 7 and 8, both for the subsystems and their interconnection. Figure 7 shows the voltages for the CIL in the three different scenarios. At $\mathrm{t}=0$, the load is equal to $100 \Omega$, amd it reduces to $50 \Omega$ at $\mathrm{t}=10 \mathrm{~s}$; the reference changes from $180 \mathrm{~V}$ to $200 \mathrm{~V}$ at $\mathrm{t}=3 \mathrm{~s}$. Figure 7 shows that the different controls correctly track the references. Small perturbations at $\mathrm{t}=4,8,12$ and $13 \mathrm{~s}$ match with the variations in other nodes. In Figure 8, the power profiles for the CPL are shown. As expected, the case considering full information is the one with the smaller perturbations. The trajectories converge to the operating points according to each power and voltage level, consistently with the power profile.

Figure 9 shows the currents of each supercapacitor in the considered cases: the associated energy levels are shown in Figure 10. As the values for $\gamma_{l}$ are identical, the current sharing mechanism forces the fast devices to contribute equally to the voltage stabilization when either full or partial information sharing is considered, . Instead, when no information is shared, the voltage stabilization participation depends mainly on the different value of the resistances in the connecting cables and on the size of the supercapacitors. This results in different
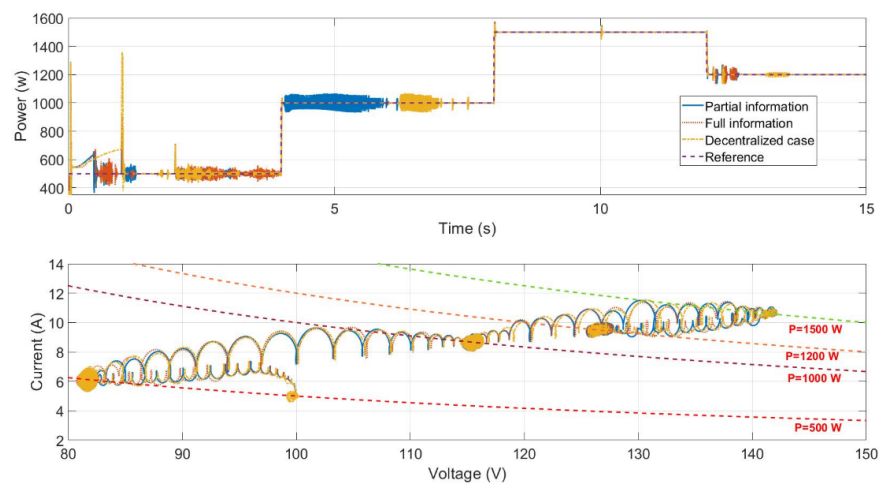

Fig. 8. Case I: a) Power in CPL with respect to the reference (dotted violet line) in case of partial information (blue line), full information (dotted red line) and no information sharing (dotted yellow line). b) Trajectories in the CPL.
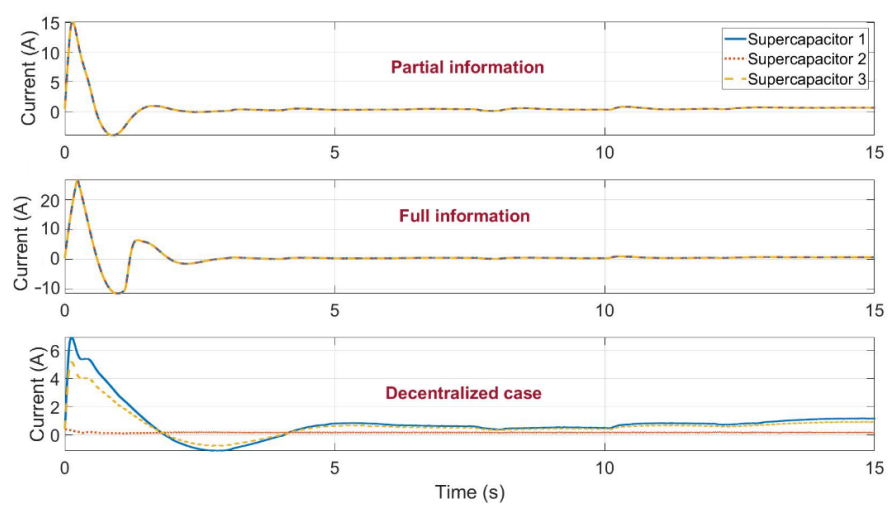

Fig. 9. Case I: The currents in the supercapacitors in the considered situations of information sharing. The current of supercapacitor 1 is the blue line, while the ones of supercapacitors 2 and 3 are the dotted red and dotted yellow lines, respectively.

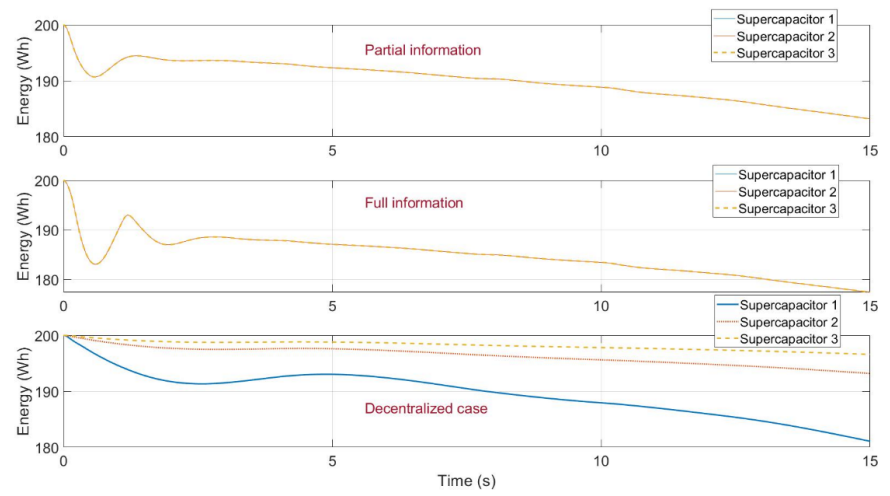

Fig. 10. Case I: The energy in the supercapacitors in the considered situations of information sharing. The energy of supercapacitor 1 is the blue line, while the ones of supercapacitors 2 and 3 are the dotted red and dotted yellow lines, respectively. 
energy profiles for the decentralized case.

The different control strategies successfully reach the target to control the DC grid voltage under adverse circumstances.

\section{B. Case II: different values for $\gamma_{l}$}

In this case, the supercapacitors have different values for the gains $\gamma_{l}: \gamma_{3}=0.3$, while $\gamma_{5}=\gamma_{7}=0.35$. Consequently, supercapacitor 1 (in node 3) will contribute less with respect to supercapacitors 2 (in node 5) and 3 (in node 7) to voltage regulation. Since these gains are not involved in the decentralized case, the comparison is performed between only the two information sharing cases. The same load variations of Case I are considered.

Figure 11 shows the DC voltage for both full and partial information cases. In both situations the controllers perform well. The voltage does not exceed the admissible considered tolerance $( \pm 10 \%)$, and the most critical error is due to the initial conditions. The more complete knowledge of the system in the full information case results in a shorter transient and a reduced peak.

Figure 12 shows the currents of each supercapacitor: from the choice of $\gamma_{l}$, the currents of supercapacitors 2 and 3 are equal and have different magnitude with respect to the one of supercapacitor 1 in both cases of full information sharing or partial one. As a result, the energy levels are different, as shown in Figure 13. In the full information case, where a unique solution is possible, the set of supercapacitors perform the same needed discharging action. The different evolution of the currents is highlighted by the different energy levels of supercapacitors 2 and 3 with respect to supercapacitor 1 . Instead, in the partial information case, where multiple possible solutions exist, the different evolution is demonstrated by the fact that supercapacitor 1 is charging while supercapacitors 2 and 3 are discharging. This situation corresponds to Section VI-C, where long-term energy strategies may underperform in the partial information case with respect to the full information one, due to inefficient power exchange among the fast devices. This situation does not impact stability (and hence, it is not considered in this paper), and a dedicated control at a higher level will be investigated in future publications. However, the proposed control laws allow engineers to choose between performance (e.g. of voltage transients or efficient power exchange) and needed communication exchange by selecting an appropriate trade-off. For lack of space, the entire set of dynamics is not shown here. Nevertheless, for sake of completeness, Figure 14 shows the currents of the supercapacitors tracking their reference trajectories. The PV and battery power outputs are introduced in Fig. 4 and 5. The control inputs of the DC/DC converters connected to the three supercapacitors are shown in Fig. 15 and 16.

The distributed coupled control action maintain the grid voltage around the desired equilibrium point, as shown in Fig. 6 and 11.

\section{CONCLUSIONS}

In this paper, we introduced a constructive ISS-Lyapunovbased current-sharing control framework for a DC microgrid

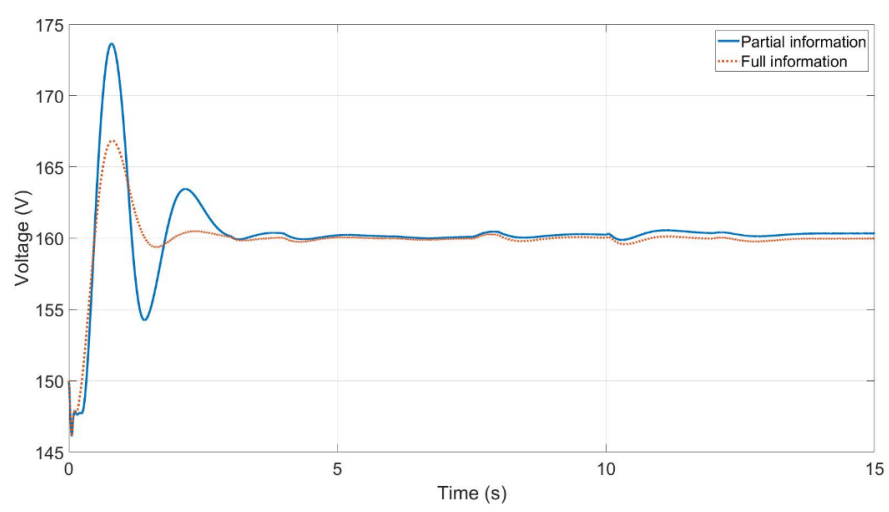

Fig. 11. Case II: The voltage of the DC grid in case of partial information (blue line) and full information (dotted red line).
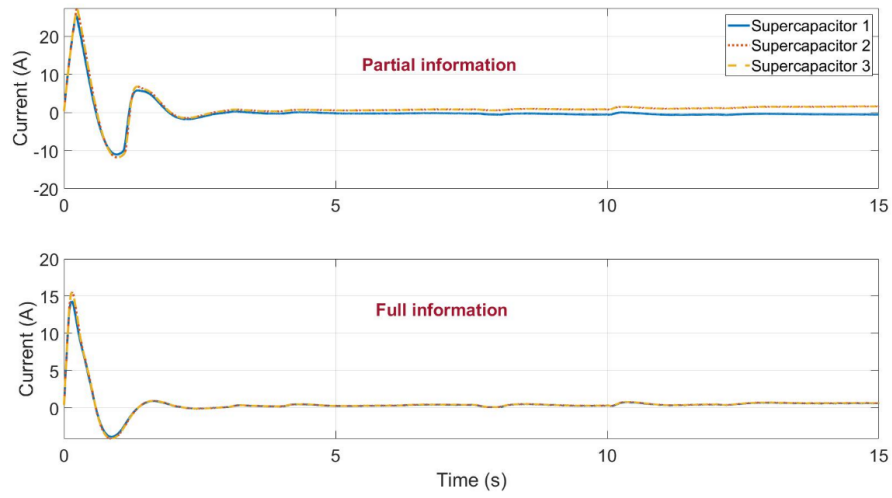

Fig. 12. Case II: The currents in the supercapacitors for the information sharing case. The current of supercapacitor 1 is the blue line, while the ones of supercapacitors 2 and 3 are the dotted red and dotted yellow lines, respectively.
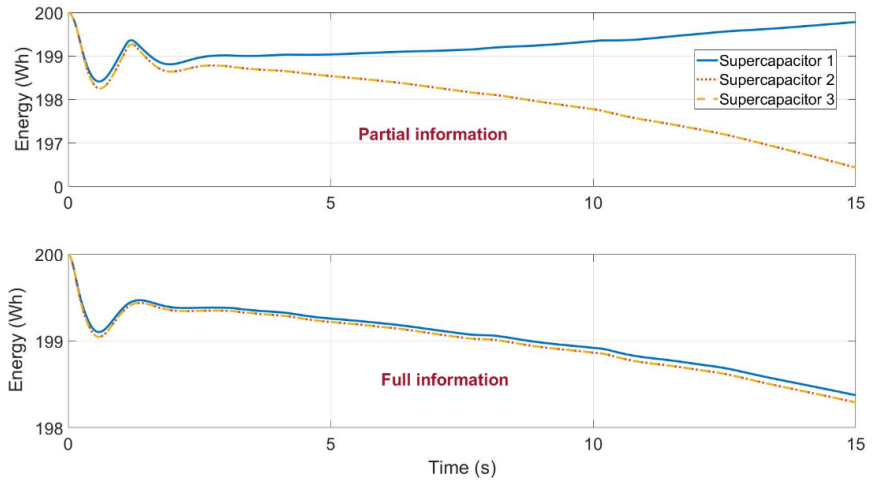

Fig. 13. Case II: The energy in the supercapacitors for the information sharing case. The energy of supercapacitor 1 is the blue line, while the ones of supercapacitors 2 and 3 are the dotted red and dotted yellow lines, respectively. 

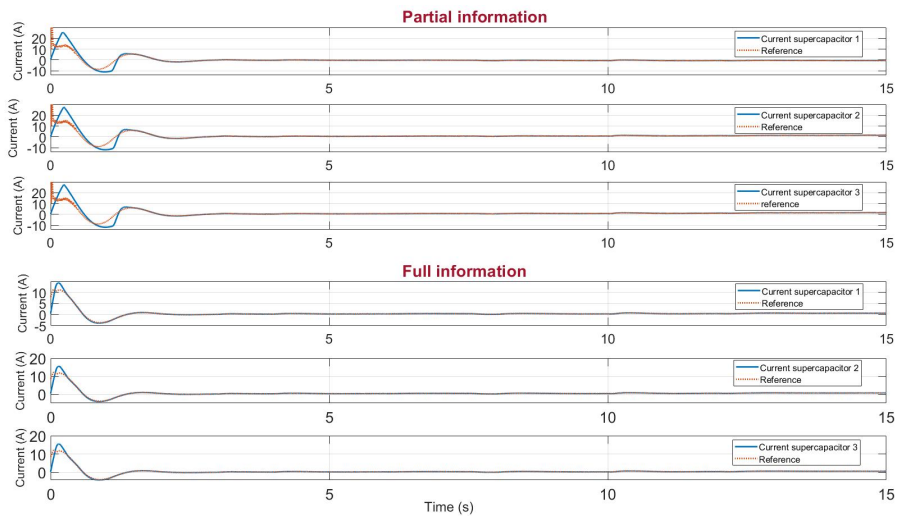

Fig. 14. Case II: The currents in the supercapacitors of Fig. 12 with respect to their references.

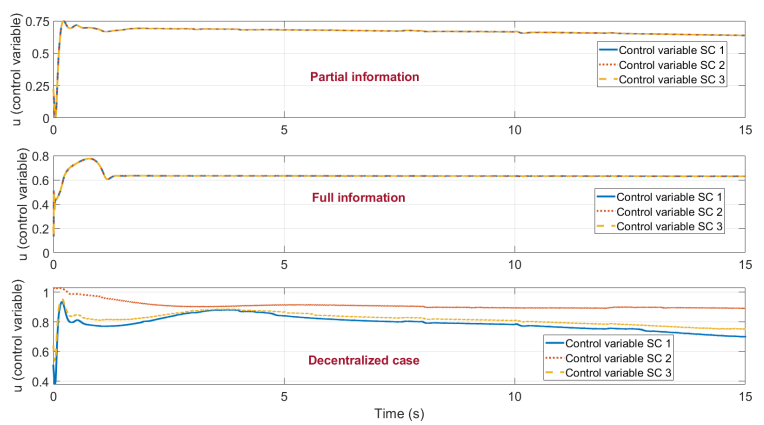

Fig. 15. Case I: The control inputs for the information sharing case. The input of supercapacitor 1 is the blue line, while the ones of supercapacitors 2 and 3 are the dotted red and dotted yellow lines, respectively.
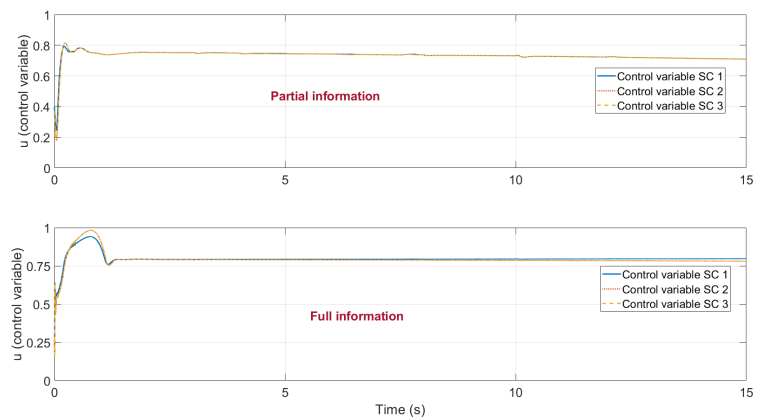

Fig. 16. Case II: The control inputs for the information sharing case. The input of supercapacitor 1 is the blue line, while the ones of supercapacitors 2 and 3 are the dotted red and dotted yellow lines, respectively. consisting of renewables, a HESS composed by two kinds of storage devices, DC loads and their connected step-up or step-down converter devices. The DC microgrid is decomposed in two subsystems with different targets, to provide a desired amount of power to uncontrolled bounded loads while ensuring a given grid-voltage value. A Lyapunov-based stability analysis is carried out for the system describing the interconnection by composing the stability analysis performed on each subsystems. The Lyapunov function is used to ensure the appropriate power exchange between the devices providing voltage regulation in a current sharing mode (similar to a fast droop control), assuming that enough power is available for long term stability. Different information scenarios are investigated. Their impact on the stability of the system are discussed. Finally, a set of simulation experiments shows the efficiency of the proposed control action.

The results presented here are general and valid for any kind of DC microgrid with a star topology consisting of step-up or step-down DC/DC converters with the objective of using renewable power to feed the loads while ensuring voltage regulation. The control method can be used in Plug\&Play mode thus allowing to scale to larger dimensions. Future work will extend the proposed framework to meshed microgrids and will focus on solutions to the multiple solution problem in the partially distributed case.

\section{REFERENCES}

[1] L. E. Zubieta, "Are microgrids the future of energy?: DC microgrids from concept to demonstration to deployment," IEEE Electrification Magazine, vol. 4, no. 2, pp. 37-44, June 2016.

[2] E. Rodriguez-Diaz, F. Chen, J. C. Vasquez, J. M. Guerrero, R. Burgos, and D. Boroyevich, "Voltage-level selection of future two-level lvdc distribution grids: A compromise between grid compatibiliy, safety, and efficiency," IEEE Electrification Magazine, vol. 4, no. 2, pp. 20-28, June 2016.

[3] U. Manandhar, N. R. Tummuru, S. K. Kollimalla, A. Ukil, G. H. Beng, and K. Chaudhari, "Validation of faster joint control strategy for battery- and supercapacitor-based energy storage system," IEEE Transactions on Industrial Electronics, vol. 65, no. 4, pp. 3286-3295, April 2018.

[4] A. Iovine, S. B. Siad, G. Damm, E. De Santis, and M. D. Di Benedetto, "Nonlinear Control of a DC MicroGrid for the Integration of Photovoltaic Panels," IEEE Transactions on Automation Science and Engineering, vol. 14, no. 2, pp. 524535, April 2017.

[5] R. F. Bastos, T. Dragicevic, J. M. Guerrero, and R. Q. Machado, "Decentralized control for renewable DC Microgrid with composite energy storage system and UC voltage restoration connected to the grid," in IECON 2016 - 42nd Annual Conference of the IEEE Industrial Electronics Society, Oct 2016, pp. 20162021.

[6] D. Olivares, A. Mehrizi-Sani, A. Etemadi, C. Canizares, R. Iravani, M. Kazerani, A. Hajimiragha, O. Gomis-Bellmunt, M. Saeedifard, R. Palma-Behnke, G. Jimenez-Estevez, and N. Hatziargyriou, "Trends in microgrid control," IEEE Transactions on Smart Grid, vol. 5, no. 4, pp. 1905-1919, July 2014.

[7] M. Farrokhabadi, C. A. Cañizares, J. W. Simpson-Porco, E. Nasr, L. Fan, P. A. Mendoza-Araya, R. Tonkoski, U. Tamrakar, N. Hatziargyriou, D. Lagos, R. W. Wies, M. Paolone, M. Liserre, L. Meegahapola, M. Kabalan, A. H. Hajimiragha, D. Peralta, M. A. Elizondo, K. P. Schneider, F. K. Tuffner, and J. Reilly, "Microgrid stability definitions, analysis, and 
examples," IEEE Transactions on Power Systems, vol. 35, no. 1, pp. 13-29, 2020.

[8] P. Kundur, J. Paserba, V. Ajjarapu, G. Andersson, A. Bose, C. Canizares, N. Hatziargyriou, D. Hill, A. Stankovic, C. Taylor, T. Van Cutsem, and V. Vittal, "Definition and classification of power system stability IEEE/CIGRE joint task force on stability terms and definitions," IEEE Transactions on Power Systems, vol. 19, no. 3, Aug 2004.

[9] N. Hatziargyriou, J. V. Milanovic, C. Rahmann, V. Ajjarapu, C. Canizares, I. Erlich, D. Hill, I. Hiskens, I. Kamwa, B. Pal, P. Pourbeik, J. J. Sanchez-Gasca, A. M. Stankovic, T. Van Cutsem, V. Vittal, and C. Vournas, "Definition and classification of power system stability revisited extended," IEEE Transactions on Power Systems, pp. 1-1, 2020.

[10] L. Meng, Q. Shafiee, G. F. Trecate, H. Karimi, D. Fulwani, $\mathrm{X}$. Lu, and J. M. Guerrero, "Review on Control of DC Microgrids and Multiple Microgrid Clusters," IEEE Journal of Emerging and Selected Topics in Power Electronics, vol. 5, no. 3, pp. 928-948, Sept 2017.

[11] J. W. Simpson-Porco, F. Dörfler, and F. Bullo, "Voltage collapse in complex power grids," Nature Communications, vol. 7, no. 10790, Feb 2016.

[12] J. Zhao and F. Dorfler, "Distributed control and optimization in DC microgrids," Automatica, vol. 61, pp. 18 - 26, 2015.

[13] C. De Persis, E. R. Weitenberg, and F. Dörfler, "A power consensus algorithm for DC microgrids," Automatica, vol. 89, pp. $364-375,2018$.

[14] J. Liu, W. Zhang, and G. Rizzoni, "Robust Stability Analysis of DC Microgrids With Constant Power Loads," IEEE Transactions on Power Systems, vol. 33, no. 1, pp. 851-860, Jan 2018.

[15] K. F. Krommydas and A. T. Alexandridis, "Modular Control Design and Stability Analysis of Isolated PV-Source/BatteryStorage Distributed Generation Systems," IEEE Journal on Emerging and Selected Topics in Circuits and Systems, vol. 5, no. 3, pp. 372-382, Sep. 2015.

[16] R. Delpoux, J.-F. Trégouët, J.-Y. Gauthier, and C. Lacombe, "New framework for optimal current sharing of nonidentical parallel buck converters," IEEE Transactions on Control Systems Technology, vol. 27, no. 3, pp. 1237-1243, 2019.

[17] R. Han, M. Tucci, A. Martinelli, J. M. Guerrero, and G. FerrariTrecate, "Stability Analysis of Primary Plug-and-Play and Secondary Leader-Based Controllers for DC Microgrid Clusters," IEEE Transactions on Power Systems, vol. 34, no. 3, pp. 17801800, May 2019.

[18] A. Iovine, M. Jimenez Carrizosa, G. Damm, and P. Alou, "Nonlinear Control for DC Microgrids Enabling Efficient Renewable Power Integration and Ancillary Services for AC Grids," IEEE Transactions on Power Systems, vol. 34, no. 6, pp. 5136-5146, Nov 2019.

[19] P. Lin, C. Zhang, J. Wang, C. Jin, and P. Wang, "On Autonomous Large-Signal Stabilization for Islanded Multibus DC Microgrids: A Uniform Nonsmooth Control Scheme," IEEE Transactions on Industrial Electronics, vol. 67, no. 6, pp. $\overline{4600-}$ 4612, 2020.

[20] M. Baranwal, A. Askarian, S. Salapaka, and M. Salapaka, "A Distributed Architecture for Robust and Optimal Control of DC Microgrids," IEEE Transactions on Industrial Electronics, vol. 66, no. 4, pp. 3082-3092, April 2019.

[21] P. Lin, C. Zhang, P. Wang, and J. Xiao, "A Decentralized Composite Controller for Unified Voltage Control With Global System Large-Signal Stability in DC Microgrids," IEEE

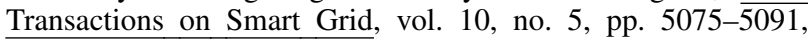
2019.

[22] M. Tucci, L. Meng, J. M. Guerrero, and G. Ferrari-Trecate, "Stable current sharing and voltage balancing in DC microgrids: A consensus-based secondary control layer," Automatica, vol. 95, pp. $1-13,2018$.

[23] M. Cucuzzella, S. Trip, C. De Persis, X. Cheng, A. Ferrara, and A. van der Schaft, "A Robust Consensus Algorithm for Current Sharing and Voltage Regulation in DC Microgrids," IEEE Transactions on Control Systems Technology, vol. 27, no. 4, pp. 1583-1595, July 2019.

[24] S. Trip, M. Cucuzzella, X. Cheng, and J. Scherpen, "Distributed Averaging Control for Voltage Regulation and Current Sharing in DC Microgrids," IEEE Control Systems Letters, vol. 3, no. 1, pp. 174-179, Jan 2019.

[25] A. Iovine, T. Rigaut, G. Damm, E. De Santis, and M. D. Di Benedetto, "Power management for a DC MicroGrid integrating renewables and storages," Control Engineering Practice, vol. 85, pp. 59 - 79, 2019.

[26] H. K. Khalil, Nonlinear systems; 3rd ed. Upper Saddle River, NJ: Prentice-Hall, 2002.

[27] M. Krstic, P. V. Kokotovic, and I. Kanellakopoulos, Nonlinear and Adaptive Control Design, 1st ed. New York, NY, USA: John Wiley \& Sons, Inc., 1995.

[28] B. Charlet, J. Lévine, and R. Marino, "On dynamic feedback linearization," Systems \& Control Letters, vol. 13, no. 2, pp. $143-151,1989$.

[29] P. Rouchon, "Necessary condition and genericity of dynamic feedback linearization,” J. Math. Systems Estim. Control, vol. 4, no. 2, pp. 257-260, 1994.

[30] E. D. Sontag, "Smooth stabilization implies coprime factorization," IEEE Transactions on Automatic Control, vol. 34, no. 4, pp. 435-443, April 1989.

[31] E. D. Sontag, Input to State Stability: Basic Concepts and Results. Berlin, Heidelberg: Springer Berlin Heidelberg, 2008, pp. 163-220.

[32] P. Pepe and H. Ito, "On Saturation, Discontinuities, and Delays, in iISS and ISS Feedback Control Redesign," IEEE Transactions on Automatic Control, vol. 57, no. 5, pp. 1125-1140, May 2012.

[33] Z.-P. Jiang, A. R. Teel, and L. Praly, "Small-gain theorem for ISS systems and applications," Mathematics of Control, Signals and Systems, vol. 7, pp. 95-120, 1994.

[34] G. C. Konstantopoulos and A. T. Alexandridis, "Generalized nonlinear stabilizing controllers for hamiltonian-passive systems with switching devices," IEEE Transactions on Control Systems Technology, vol. 21, no. 4, pp. 1479-1488, July 2013.

[35] L. Aolaritei, D. Lee, T. L. Vu, and K. Turitsyn, “A Robustness Measure of Transient Stability Under Operational Constraints in Power Systems," IEEE Control Systems Letters, vol. 2, no. 4, pp. 803-808, 2018.

[36] P. C. Papageorgiou and A. T. Alexandridis, "Controlled impedance-admittance-torque nonlinear modeling and analysis of modern power systems," Energies, vol. 13, no. 10, 2020. [Online]. Available: https://www.mdpi.com/1996-1073/13/10/ 2461

[37] J. Ferguson, M. Cucuzzella, and J. M. A. Scherpen, "Exponential Stability and Local ISS for DC Networks," IEEE Control Systems Letters, vol. 5, no. 3, pp. 893-898, 2021.

[38] A. Iovine, G. Damm, E. De Santis, M. D. Di Benedetto, L. Galai-Dol, and P. Pepe, "Voltage Stabilization in a DC MicroGrid by an ISS-like Lyapunov Function implementing Droop Control," in 2018 European Control Conference (ECC), June 2018, pp. 1130-1135.

[39] M. Forouzesh, Y. P. Siwakoti, S. A. Gorji, F. Blaabjerg, and B. Lehman, "Step-Up DC-DC Converters: A Comprehensive Review of Voltage-Boosting Techniques, Topologies, and Applications," IEEE Transactions on Power Electronics, vol. 32, no. 12, pp. 9143-9178, Dec 2017.

[40] P. Nahata, R. Soloperto, M. Tucci, A. Martinelli, and G. FerrariTrecate, "A passivity-based approach to voltage stabilization in DC microgrids with ZIP loads," Automatica, vol. 113, p. 108770, 2020.

[41] R. Han, H. Wang, Z. Jin, L. Meng, and J. M. Guerrero, "Compromised Controller Design for Current Sharing and Voltage Regulation in DC Microgrid," IEEE Transactions on Power Electronics, vol. 34, no. 8, pp. 8045-8061, Aug 2019. 
[42] F. Perez, A. Iovine, G. Damm, L. Galai-Dol, and P. F. Ribeiro, "Stability Analysis of a DC MicroGrid for a Smart Railway Station Integrating Renewable Sources," IEEE Transactions on Control Systems Technology, vol. 28, no. 5, pp. 1802-1816, 2020.

[43] Z.-P. Jiang, I. M. Mareels, and Y. Wang, "A Lyapunov formulation of the nonlinear small-gain theorem for interconnected ISS systems," Automatica, vol. 32, no. 8, pp. 1211 - 1215, 1996.

[44] N. Mohan, T. M. Undeland, and W. P. Robbins, Power electronics. Wiley, 1995.

[45] M. D. Seeman and S. R. Sanders, "Analysis and optimization of switched-capacitor dc-dc converters," IEEE Transactions on Power Electronics, vol. 23, no. 2, pp. 841-851, 2008.

[46] S. R. Sanders and G. C. Verghese, "Synthesis of averaged circuit models for switched power converters," in IEEE International Symposium on Circuits and Systems, 1990, pp. 679-683 vol.1.

[47] N. Yang, B. Nahid-Mobarakeh, F. Gao, D. Paire, A. Miraoui, and W. Liu, "Modeling and stability analysis of multi-time scale DC microgrid," Electric Power Systems Research, vol. 140, pp. $906-916,2016$.

[48] J. R. Winkelman, J. H. Chow, J. J. Allemong, and P. V. Kokotovic, "Multi-time-scale analysis of a power system," Automatica, vol. 16, no. 1, pp. $35-43,1980$.

[49] P. V. Kokotovic, H. K. Khalil, and J. O'Reilly, Singular perturbation methods in control. Academic Press, 1986.

[50] Y. Chen, M. Jimenez Carrizosa, G. Damm, F. LamnabhiLagarrigue, M. Li, and Y. Li, "Control-induced time-scale separation for multiterminal high-voltage direct current systems using droop control," IEEE Transactions on Control Systems Technology, pp. 1-17, 2019.

[51] J. W. Kimball and P. T. Krein, "Singular Perturbation Theory for DC-DC Converters and Application to PFC Converters," IEEE Transactions on Power Electronics, vol. 23, no. 6, pp. $\overline{2970-}$ 2981, Nov 2008.

[52] I. Karafyllis and Z.-P. Jiang, Stability and Stabilization of Nonlinear Systems. Springer Science Business Media, 2011.

[53] D. Zonetti, R. Ortega, and A. Benchaib, "Modeling and control of HVDC transmission systems from theory to practice and back," Control Engineering Practice, vol. 45, pp. 133 - 146, 2015.

\section{APPENDIX}

\section{A. Calculations for $W$}

Here we leverage the construction of the Lyapunov function $W$ when proving that the control inputs $u_{l}$ in (15) and (16) with respect to the references $z_{l}$ in (18), (21), (22) and $r_{l}$ in (23) and (26) stabilize the system in (11). We recall the error states $\tilde{v}_{l}^{b}$ and $\tilde{i}_{l}$ as

$$
\tilde{v}_{l}^{b}=v_{l}^{b}-z_{l}, \quad \tilde{i}_{l}=i_{l}-r_{l}
$$

We begin by computing the time derivative of the storage function $W^{\text {bus }}\left(v_{j}^{b}, v_{\text {bus }}\right)$ in (28), i.e. $\dot{W}^{\text {bus }}\left(v_{j}^{b}, v_{b u s}\right)$ in (29):

$$
\begin{aligned}
\dot{W}^{\text {bus }}= & \sum_{j \in \mathcal{I}_{\eta}} v_{j}^{b}\left[-g_{j}^{b} v_{j}^{b}+\frac{v_{\text {bus }}-v_{j}^{b}}{R_{j}^{b}}+i_{j}\left(1-u_{j} \zeta_{j}\right)\right] \\
& +v_{\text {bus }}\left[\sum_{j \in \mathcal{I}_{\eta}} \frac{v_{j}^{b}-v_{\text {bus }}}{R_{j}^{b}}+\sum_{l \in \mathcal{I}_{\phi}} \frac{\tilde{v}_{l}^{b}+z_{l}-v_{\text {bus }}}{R_{l}^{b}}\right] \\
= & -\sum_{j \in \mathcal{I}_{\eta}} \frac{\left(v_{j}^{b}-v_{b u s}\right)^{2}}{R_{j}^{b}}+\sum_{j \in \mathcal{I}_{\eta}} v_{j}^{b}\left[i_{j}\left(1-u_{j} \zeta_{j}\right)-g_{j}^{b} v_{j}^{b}\right] \\
& +v_{\text {bus }} \sum_{l \in \mathcal{I}_{\phi}} \frac{1}{R_{l}}\left[z_{l}-v_{\text {bus }}\right]+v_{\text {bus }} \sum_{l \in \mathcal{I}_{\phi}} \frac{1}{R_{l}} \tilde{v}_{l}^{b} \\
= & -\Psi_{\mathcal{I}_{\eta}, v_{\text {bus }}}+\Psi_{\mathcal{I}_{\eta}}+v_{\text {bus }} \sum_{l \in \mathcal{I}_{\phi}} \frac{z_{l}-v_{\text {bus }}}{R_{l}}+v_{\text {bus }} \sum_{l \in \mathcal{I}_{\phi}} \frac{\tilde{v}_{l}^{b}}{R_{l}}
\end{aligned}
$$

where $\Psi_{\mathcal{I}_{\eta}, v_{b u s}}$ and $\Psi_{\mathcal{I}_{\eta}}$ are defined in (30) and (17).

According to the considered information scenario and the related choice for $z_{l}, \dot{W}^{\text {bus }}$ in (53) is defined as:

- Distributed control case: complete-information of state and control variables

$$
\begin{gathered}
\dot{W}^{\text {bus }}=-\Psi_{\mathcal{I}_{\eta}, v_{b u s}}-\sum_{l \in \mathcal{I}_{\phi}}\left(\frac{1}{R_{l}}+\gamma_{l} K_{l}\right) v_{\text {bus }}^{2} \\
+\sum_{l \in \mathcal{I}_{\phi}}\left(\frac{1}{R_{l}}+\gamma_{l} K_{l}\right) \bar{v}_{\text {bus }}^{2}+v_{\text {bus }} \sum_{l \in \mathcal{I}_{\phi}} \frac{1}{R_{l}} \tilde{v}_{l}^{b}
\end{gathered}
$$

- Partially distributed control case: partial-information of state and control variables

$$
\begin{aligned}
& \dot{W}^{\text {bus }}=-\Psi_{\mathcal{I}_{\eta}, v_{\text {bus }}}-\sum_{l \in \mathcal{I}_{\phi}}\left(\frac{1}{R_{l}}+\gamma_{l} K_{l}\right) v_{\text {bus }}^{2}+\Psi_{\max } \\
& +\sum_{l \in \mathcal{I}_{\phi}}\left(\frac{1}{R_{l}}+\gamma_{l} K_{l}\right) \bar{v}_{\text {bus }}^{2}+v_{\text {bus }} \sum_{l \in \mathcal{I}_{\phi}} \frac{1}{R_{l}} \tilde{v}_{l}^{b}
\end{aligned}
$$

\section{- Decentralized control case: no-information}

$$
\begin{aligned}
& \dot{W}^{\text {bus }}=-\Psi_{\mathcal{I}_{\eta}, v_{\text {bus }}}-\sum_{l \in \mathcal{I}_{\phi}}\left(\frac{1}{R_{l}}+K_{l}\right) v_{\text {bus }}^{2}+\Psi_{\max } \\
& +\sum_{l \in \mathcal{I}_{\phi}}\left(\frac{1}{R_{l}}+K_{l}\right) \bar{v}_{\text {bus }}^{2}+v_{\text {bus }} \sum_{l \in \mathcal{I}_{\phi}} \frac{1}{R_{l}} \tilde{v}_{l}^{b}
\end{aligned}
$$

From here on, we refer to the distributed case without loss of generality. We introduce the following Lyapunov function $W^{\text {bus,b }}$, and describe the step-down and step-up cases:

$$
W^{b u s, b}=W^{b u s}+\sum_{l \in \mathcal{I}_{\phi}} \frac{C_{l}^{b}}{2}\left(\tilde{v}_{l}^{b}\right)^{2}
$$


1) step-down case, a) in Table I: The time derivative $\dot{W}^{b u s, b}$ of $W^{b u s, b}$ in (57) is:

$$
\begin{aligned}
& \dot{W}^{\text {bus }, b}=\dot{W}^{\text {bus }}+\sum_{l \in \mathcal{I}_{\phi}} \tilde{v}_{l}^{b} \dot{\tilde{v}}_{l}^{b}=-\Psi_{\mathcal{I}_{\eta}, v_{b u s}} \\
& -\sum_{l \in \mathcal{I}_{\phi}}\left(\frac{1}{R_{l}}+\gamma_{l} K_{l}\right)\left(v_{b u s}^{2}-\bar{v}_{b u s}^{2}\right)+v_{b u s} \sum_{l \in \mathcal{I}_{\phi}} \frac{1}{R_{l}} \tilde{v}_{l}^{b} \\
& +\sum_{l \in \mathcal{I}_{\phi}} \tilde{v}_{l}^{b}\left[-\left(g_{l}^{b}+\frac{1}{R_{l}}\right)\left(\tilde{v}_{l}^{b}+z_{l}\right)+\frac{1}{R_{l}} v_{b u s}\right] \\
& +\sum_{l \in \mathcal{I}_{\phi}} \tilde{v}_{l}^{b}\left[\left(\tilde{i}_{l}+r_{l}\right)-C_{l} \dot{z}_{l}\right] \\
& =-\Psi_{\mathcal{I}_{\eta}, v_{b u s}}-\sum_{l \in \mathcal{I}_{\phi}}\left(\frac{1}{R_{l}}+\gamma_{l} K_{l}\right)\left(v_{b u s}^{2}-\bar{v}_{b u s}^{2}\right) \\
& -\sum_{l \in \mathcal{I}_{\phi}}\left(g_{l}^{b}+\frac{1}{R_{l}}\right)\left(\tilde{v}_{l}^{b}\right)^{2}+\sum_{l \in \mathcal{I}_{\phi}} \tilde{v}_{l}^{b} \tilde{i}_{l} \\
& +\sum_{l \in \mathcal{I}_{\phi}} \tilde{v}_{l}^{b}\left[-\left(g_{l}^{b}+\frac{1}{R_{l}}\right) z_{l}+\frac{2}{R_{l}} v_{b u s}+r_{l}-C_{l} \dot{z}_{l}\right] .
\end{aligned}
$$

The definition of $r_{l}$, as in (23), i.e.

$$
r_{l}=\left(g_{l}^{b}+\frac{1}{R_{l}^{b}}\right) z_{l}-\frac{2}{R_{l}^{b}} v_{b u s}+C_{l}^{b} \dot{z}_{l}-K_{l}^{b}\left(v_{l}^{b}-z_{l}\right),
$$

modifies $\dot{W}^{\text {bus }, b}$ in (58) to become

$$
\begin{aligned}
& \dot{W}^{\text {bus }, b}=-\Psi_{\mathcal{I}_{\eta}, v_{b u s}}-\sum_{l \in \mathcal{I}_{\phi}}\left(\frac{1}{R_{l}}+\gamma_{l} K_{l}\right)\left(v_{b u s}^{2}-\bar{v}_{b u s}^{2}\right) \\
& -\sum_{l \in \mathcal{I}_{\phi}}\left[\left(g_{l}^{b}+\frac{1}{R_{l}}\right)+K_{l}^{b}\right]\left(\tilde{v}_{l}^{b}\right)^{2}+\sum_{l \in \mathcal{I}_{\phi}} \tilde{v}_{l}^{b} \tilde{i}_{l} .
\end{aligned}
$$

We introduce the storage function $W^{b u s, b, i}$ as

$$
W^{b u s, b, i}=W^{b u s, b}+W^{i}=W^{b u s, b}+\sum_{l \in \mathcal{I}_{\phi}} \frac{L_{l}}{2}\left(\tilde{i}_{l}\right)^{2}
$$

and compute its time derivative:

$$
\begin{aligned}
& \dot{W}^{\text {bus }, b, i}=-\Psi_{\mathcal{I}_{\eta}, v_{b u s}}-\sum_{l \in \mathcal{I}_{\phi}}\left(\frac{1}{R_{l}}+\gamma_{l} K_{l}\right)\left(v_{b u s}^{2}-\bar{v}_{b u s}^{2}\right) \\
& -\sum_{l \in \mathcal{I}_{\phi}}\left[\left(g_{l}^{b}+\frac{1}{R_{l}}\right)+K_{l}^{b}\right]\left(\tilde{v}_{l}^{b}\right)^{2}+\sum_{l \in \mathcal{I}_{\phi}} \tilde{i}_{l}\left[\tilde{v}_{l}^{b}+\dot{\tilde{i}}_{l}\right] \\
& =-\Psi_{\mathcal{I}_{\eta}, v_{b u s}}-\sum_{l \in \mathcal{I}_{\phi}}\left(\frac{1}{R_{l}}+\gamma_{l} K_{l}\right)\left(v_{\text {bus }}^{2}-\bar{v}_{b u s}^{2}\right) \\
& -\sum_{l \in \mathcal{I}_{\phi}}\left[\left(g_{l}^{b}+\frac{1}{R_{l}}\right)+K_{l}^{b}\right]\left(\tilde{v}_{l}^{b}\right)^{2} \\
& +\sum_{l \in \mathcal{I}_{\phi}} \tilde{i}_{l}\left[\tilde{v}_{l}^{b}-\left(\tilde{v}_{l}^{b}+z_{l}\right)-\left(R_{l}^{s}+R_{l}^{r}\right)\left(\tilde{i}_{l}+r_{l}\right)+v_{l}^{d} u_{l}-L_{l} \dot{r}_{l}\right] \\
& =-\Psi_{\mathcal{I}_{\eta}, v_{b u s}}-\sum_{l \in \mathcal{I}_{\phi}}\left(\frac{1}{R_{l}}+\gamma_{l} K_{l}\right)\left(v_{b u s}^{2}-\bar{v}_{b u s}^{2}\right) \\
& -\sum_{l \in \mathcal{I}_{\phi}}\left[\left(g_{l}^{b}+\frac{1}{R_{l}}\right)+K_{l}^{b}\right]\left(\tilde{v}_{l}^{b}\right)^{2}-\sum_{l \in \mathcal{I}_{\phi}}\left(R_{l}^{s}+R_{l}^{r}\right)\left(\tilde{i}_{l}\right)^{2} \\
& +\sum_{l \in \mathcal{I}_{\phi}} \tilde{i}_{l}\left[-z_{l}-\left(R_{l}^{s}+R_{l}^{r}\right) r_{l}+v_{l}^{d} u_{l}-L_{l} \dot{r}_{l}\right] .
\end{aligned}
$$

The choice of $u_{l}$ as in (15), i.e.

$$
u_{l}=\frac{1}{v_{l}^{d}}\left[\left(R_{l}^{s}+R^{r}\right){ }_{l} r_{l}+z_{l}+L_{l} \dot{r}_{l}-K_{l}^{i} \tilde{i}_{l}\right],
$$

provides the final form of $\dot{W}^{b u s, b, i}$ as

$$
\begin{aligned}
\dot{W}^{b u s, b, i}= & -\Psi_{\mathcal{I}_{\eta}, v_{b u s}}-\sum_{l \in \mathcal{I}_{\phi}}\left(\frac{1}{R_{l}}+\gamma_{l} K_{l}\right)\left(v_{b u s}^{2}-\bar{v}_{b u s}^{2}\right) \\
& -\sum_{l \in \mathcal{I}_{\phi}}\left[\left(g_{l}^{b}+\frac{1}{R_{l}}\right)+K_{l}^{b}\right]\left(\tilde{v}_{l}^{b}\right)^{2} \\
& -\sum_{l \in \mathcal{I}_{\phi}}\left[\left(R_{l}^{s}+R_{l}^{r}\right)+K_{l}^{i}\right] .\left(\tilde{i}_{l}\right)^{2} .
\end{aligned}
$$

Equation (64) can be rewritten as

$$
\begin{aligned}
& \dot{W}^{\text {bus }, b, i}=-\Psi_{\mathcal{I}_{\eta}, v_{b u s}}-\sum_{l \in \mathcal{I}_{\phi}}\left(\frac{1}{R_{l}}+\gamma_{l} K_{l}\right)\left(v_{b u s}^{2}-\bar{v}_{b u s}^{2}\right) \\
& -\sum_{l \in \mathcal{I}_{\phi}}\left[\begin{array}{lll}
\tilde{v}_{l}^{b} & \tilde{i}_{l}
\end{array}\right] \underbrace{\left[\begin{array}{cc}
\left(g_{l}^{b}+\frac{1}{R_{l}}\right)+K_{l}^{b} & 0 \\
0 & \left(R_{l}^{s}+R_{l}^{r}\right)+K_{l}^{i}
\end{array}\right]}_{Q_{l}^{A}}\left[\begin{array}{c}
\tilde{v}_{l}^{b} \\
\tilde{i}_{l}
\end{array}\right] .
\end{aligned}
$$

2) step-up case, b) in Table I: We:

- introduce a control input $u_{l}$ such that the current variable $i_{l}$ tracks a reference $r_{l}$ to be defined;

- decouple the dynamics of $i_{l}$ and $v_{l}^{b}$ using singular perturbation theory;

- use the time derivative of $r_{l}$ as dynamic controller for stabilizing $v_{l}^{b}$;

- integrate $\dot{r}_{l}$ to find the reference $r_{l}$.

To let $i_{l}$ track a desired reference $r_{l}$, we define the storage function $W^{i}$ and its time derivative:

$$
W^{i}=\sum_{l \in \mathcal{I}_{\phi}} \frac{L_{l}}{2}\left(\tilde{i}_{l}\right)^{2}
$$

$$
\dot{W}^{i}=\sum_{l \in \mathcal{I}_{\phi}} \tilde{i}_{l}\left[v^{d}-\left(R_{l}^{s}+R_{l}^{r}\right)\left(\tilde{i}_{l}+r_{l}\right)-u_{l}\left(\tilde{v}_{l}^{b}+z_{l}\right)-L_{l} \dot{r}_{l}\right] .
$$

The control input $u_{l}$ in (16) imposes a negative definite $\dot{W}^{i}$ :

$$
\begin{gathered}
u_{l}=\frac{1}{\tilde{v}_{l}^{b}+z_{l}}\left[v_{l}^{d}-\left(R_{l}^{s}+R_{l}^{r}\right) r_{l}-L_{l} \dot{r}_{l}+K_{l}^{i} \tilde{i}_{l}\right] ; \\
\dot{W}^{i}=-\sum_{l \in \mathcal{I}_{\phi}}\left[\left(R_{l}^{s}+R_{l}^{r}\right)+K_{l}^{i}\right]\left(\tilde{i}_{l}\right)^{2} .
\end{gathered}
$$

The dynamics of $\tilde{i}_{l}$ is

$$
\dot{i}_{l}=-\left[\frac{\left(R_{l}^{s}+R_{l}^{r}\right)+K_{l}^{i}}{L_{l}}\right] \tilde{i}_{l}, \forall l \in \mathcal{I}_{\phi} .
$$

We then rewrite the equations of $i_{l}$ and $v_{l}^{b}$ as

$$
\left\{\begin{array}{l}
C_{l}^{b} \dot{v}_{l}^{b}=\frac{1}{R_{l}^{b}} v_{\text {bus }}-\left[g_{l}^{b}+\frac{1}{R_{l}^{b}}\right]\left(\tilde{v}_{l}^{b}+z_{l}\right)+\left(\tilde{i}_{l}+r_{l}\right)\left(1-u_{l}\right) \\
L_{l} \dot{i}_{l}=-\left[\left(R_{l}^{s}+R_{l}^{r}\right)+K_{l}^{i}\right] \tilde{i}_{l}
\end{array}\right.
$$

In a well-designed converter, $L_{l}$ is small enough to be chosen as the $\varepsilon$ value in the singular perturbation theory (see [49], 
[27]), i.e., the current dynamics is way faster than the voltage one. In singular perturbation standard form, the equations in (71) become

$$
\left\{\begin{array}{l}
\dot{v}_{l}^{b}=f_{v}\left(\tilde{v}_{l}^{b}, z_{l}, v_{b u s}, \tilde{i}_{l}, r_{l}\right) \\
\varepsilon \dot{i}_{l}=-\left[\left(R_{l}^{s}+R_{l}^{r}\right)+K_{l}^{i}\right] \tilde{i}_{l}
\end{array}\right.
$$

Consequently, for $\varepsilon \rightarrow 0$, we have

$$
0=-\left[\left(R_{l}^{s}+R_{l}^{r}\right)+K_{l}^{i}\right] \tilde{i}_{l}
$$

and $\tilde{i}_{l}=0$. Then, we apply control $u_{l}$ in (68) to the expression of $\tilde{v}_{l}^{b}$ considering $\tilde{i}_{l}=0$. We obtain:

$$
\begin{aligned}
\dot{\tilde{v}}_{l}^{b} & =-\left[\frac{g_{l}^{b}}{C_{l}^{b}}+\frac{1}{R_{l}^{b} C_{l}^{b}}\right]\left(\tilde{v}_{l}^{b}+z_{l}\right)+\frac{1}{R_{l}^{b} C_{l}^{b}} v_{b u s}+\frac{1}{C_{l}^{b}} r_{l} \\
& -\frac{1}{C_{l}^{b}} r_{l} \frac{1}{\tilde{v}_{l}^{b}+z_{l}}\left[v_{l}^{d}+\left(R_{l}^{s}+R_{l}^{r}\right) r_{l}\right]-\dot{z}_{l} .
\end{aligned}
$$

We then consider $W^{b u s, b}$ as in (57), and compute its time derivative $\dot{W}^{\text {bus }, b}$ :

$$
\begin{aligned}
& \dot{W}^{\text {bus }, b}=\dot{W}^{\text {bus }}+\sum_{l \in \mathcal{I}_{\phi}} \tilde{v}_{l}^{b} \dot{\tilde{v}}_{l}^{b}=-\Psi_{\mathcal{I}_{\eta}, v_{\text {bus }}} \\
& -\sum_{l \in \mathcal{I}_{\phi}}\left(\frac{1}{R_{l}}+\gamma_{l} K_{l}\right)\left(v_{\text {bus }}^{2}-\bar{v}_{\text {bus }}^{2}\right)-\sum_{l \in \mathcal{I}_{\phi}}\left(g_{l}^{b}+\frac{1}{R_{l}}\right)\left(\tilde{v}_{l}^{b}\right)^{2} \\
& +\sum_{l \in \mathcal{I}_{\phi}} \tilde{v}_{l}^{b}\left[\frac{2}{R_{l}} v_{\text {bus }}-\left(g_{l}^{b}+\frac{1}{R_{l}}\right) z_{l}+r_{l}-C_{l} \dot{z}_{l}\right] \\
& -\sum_{l \in \mathcal{I}_{\phi}} \tilde{v}_{l}^{b}\left[\frac{1}{\tilde{v}_{l}^{v}+z_{l}} r_{l}\left(v^{d}+\left(R_{l}^{s}+R_{l}^{r}\right) r_{l}\right)\right] .
\end{aligned}
$$

Consequently, we define the function $\dot{f}_{l}^{b}$ as

$$
\begin{aligned}
\dot{f}_{l}^{b}= & \frac{2}{R_{l}} v_{\text {bus }}-\left(g_{l}^{b}+\frac{1}{R_{l}}\right) z_{l}+r_{l}-C_{l} \dot{z}_{l} \\
& -\frac{1}{\tilde{v}_{l}^{v}+z_{l}} r_{l}\left(v^{d}+\left(R_{l}^{s}+R_{l}^{r}\right) r_{l}\right)=L_{f_{v}}^{1}
\end{aligned}
$$

and, by remarking that $\dot{f}_{l}^{b}$ appears in the last two lines of the inequality in (75), we rewrite equation (75) as

$$
\begin{aligned}
\dot{W}^{\text {bus }, b}= & -\Psi_{\mathcal{I}_{\eta}, v_{b u s}}-\sum_{l \in \mathcal{I}_{\phi}}\left(\frac{1}{R_{l}}+\gamma_{l} K_{l}\right)\left(v_{b u s}^{2}-\bar{v}_{b u s}^{2}\right) \\
& -\sum_{l \in \mathcal{I}_{\phi}}\left(g_{l}^{b}+\frac{1}{R_{l}}\right)\left(\tilde{v}_{l}^{b}\right)^{2}+\sum_{l \in \mathcal{I}_{\phi}} \tilde{v}_{l}^{b} \dot{f}_{l}^{b} .
\end{aligned}
$$

Therefore, we define the storage function $W^{b u s, b, f}$ as

$$
W^{b u s, b, f}=W^{b u s, b}+\frac{1}{2} \sum_{l \in \mathcal{I}_{\phi}}\left(\dot{f}_{l}^{b}\right)^{2},
$$

where the time derivative of $\dot{f}_{l}^{b}$ in (76) is

$$
\begin{aligned}
\ddot{f}_{v}= & \frac{2}{R_{l}} \dot{v}_{b u s}-\left(g_{l}^{b}+\frac{1}{R_{l}}\right) \dot{z}_{l}-C_{l} \ddot{z}_{l} \\
& -\frac{1}{\left(\tilde{v}_{l}^{v}+z_{l}\right)^{2}} r_{l}\left(\dot{\tilde{v}}_{l}^{v}+\dot{z}_{l}\right)\left(v^{d}+\left(R_{l}^{s}+R_{l}^{r}\right) r_{l}\right) \\
& +\dot{r}_{l}\left[1-\frac{v^{d}+2\left(R_{l}^{s}+R_{l}^{r}\right) r_{l}}{\tilde{v}_{l}^{v}+z_{l}}\right] \\
= & L_{f_{v}}^{2}+L_{g_{v}} \dot{r}_{l}
\end{aligned}
$$

and

$$
\begin{aligned}
\dot{W}^{b u s, b, f}= & \dot{W}^{b u s, b}+\sum_{l \in \mathcal{I}_{\phi}} \dot{f}_{l}^{b} \ddot{f}_{l}^{b} \\
= & -\Psi_{\mathcal{I}_{\eta}, v_{b u s}}-\sum_{l \in \mathcal{I}_{\phi}}\left(\frac{1}{R_{l}}+\gamma_{l} K_{l}\right)\left(v_{b u s}^{2}-\bar{v}_{b u s}^{2}\right) \\
& -\sum_{l \in \mathcal{I}_{\phi}}\left(g_{l}^{b}+\frac{1}{R_{l}}\right)\left(\tilde{v}_{l}^{b}\right)^{2}+\sum_{l \in \mathcal{I}_{\phi}} \dot{f}_{l}^{b}\left[\tilde{v}_{l}^{b}+\ddot{f}_{l}^{b}\right] .
\end{aligned}
$$

Consequently, the choice of $r_{l}$ as in (26), i.e.

$$
r_{l}(t)=\int_{0}^{t} \frac{1}{L_{g_{v}}}\left[-L_{f_{v}}^{2}-\tilde{v}_{l}^{b}-\bar{K}_{f} \dot{f}_{v}\right] d \tau
$$

allows for a negative definite $\dot{W}^{\text {bus }, b, f}$ :

$$
\begin{aligned}
& \dot{W}^{\text {bus }, b}=\dot{W}^{\text {bus }}+\sum_{l \in \mathcal{I}_{\phi}} \tilde{v}_{l}^{b} \dot{\tilde{v}}_{l}^{b} \\
& =-\Psi_{\mathcal{I}_{\eta}, v_{\text {bus }}}-\sum_{l \in \mathcal{I}_{\phi}}\left(\frac{1}{R_{l}}+\gamma_{l} K_{l}\right)\left(v_{\text {bus }}^{2}-\bar{v}_{b u s}^{2}\right) \\
& -\sum_{l \in \mathcal{I}_{\phi}}\left(g_{l}^{b}+\frac{1}{R_{l}}\right)\left(\tilde{v}_{l}^{b}\right)^{2}-\sum_{l \in \mathcal{I}_{\phi}} \bar{K}_{f}\left(\dot{f}_{l}^{b}\right)^{2} \\
& =-\Psi_{\mathcal{I}_{\eta}, v_{b u s}}-\sum_{l \in \mathcal{I}_{\phi}}\left(\frac{1}{R_{l}}+\gamma_{l} K_{l}\right)\left(v_{b u s}^{2}-\bar{v}_{b u s}^{2}\right) \\
& -\sum_{l \in \mathcal{I}_{\phi}}\left[\begin{array}{ll}
\tilde{v}_{l}^{b} & \dot{f}_{l}^{b}
\end{array}\right]^{T}\left[\begin{array}{cc}
\left(g_{l}^{b}+\frac{1}{R_{l}}\right) & 0 \\
0 & \bar{K}_{f}
\end{array}\right]\left[\begin{array}{c}
\tilde{v}_{l}^{b} \\
\dot{f}_{v}
\end{array}\right] .
\end{aligned}
$$

A storage function for the entire set of dynamics is

$$
W^{b u s, b, f, i}=W^{b u s, b, f}+W^{i}
$$

and, according to (69) and (82), its time derivative is

$\dot{W}^{b u s, b, f, i}=\dot{W}^{b u s, b, f}+\dot{W}^{i}=$

$$
\begin{aligned}
& =-\Psi_{\mathcal{I}_{\eta}, v_{b u s}}-\sum_{l \in \mathcal{I}_{\phi}}\left(\frac{1}{R_{l}}+\gamma_{l} K_{l}\right)\left(v_{b u s}^{2}-\bar{v}_{b u s}^{2}\right) \\
& -\sum_{l \in \mathcal{I}_{\phi}}\left[\begin{array}{c}
\tilde{v}_{l}^{b} \\
\dot{f}_{l}^{b} \\
\tilde{i}_{l}^{b}
\end{array}\right]^{T} \underbrace{\left[\begin{array}{ccc}
\left(g_{l}^{b}+\frac{1}{R_{l}}\right) & 0 & 0 \\
0 & \bar{K}_{f} & 0 \\
0 & 0 & \left(R_{l}^{s}+R_{l}^{r}\right)+K_{l}^{i}
\end{array}\right]}_{Q_{l}^{B}}\left[\begin{array}{c}
\tilde{v}_{l}^{b} \\
\dot{f}_{l}^{b} \\
\tilde{i}_{l}
\end{array}\right] .
\end{aligned}
$$

3) Conclusion: Given the results in Sections A1 and A2, the inequality in (45) holds with respect to the choice in (18) for $z_{l}$. The results for the choices of $z_{l}$ in (21) and (22) are similar. 